\title{
Optimal approximation of internal controls for a wave-type problem with fractional Laplacian using finite-difference method
}

\author{
Pierre Lissy * $\quad$ Ionel Rovenţa ${ }^{\dagger}$
}

September 2, 2018

\begin{abstract}
We consider a finite-difference semi-discrete scheme for the approximation of internal controls of a one-dimensional evolution problem of hyperbolic type involving the spectral fractional Laplacian. The continuous problem is controllable in arbitrary small time. However, the high frequency numerical spurious oscillations lead to a loss of the uniform (with respect to the mesh size) controllability property of the semi-discrete model in the natural setting. For all initial data in the natural energy space, if we filter the high frequencies of these initial data in an optimal way, we restore the uniform controllability property in arbitrary small time. The proof is mainly based on a (non-classic) moment method.
\end{abstract}

Keywords: fractional Laplacian, hyperbolic equations, control approximation, moment problem, biorthogonal families.

Mathematical subject codes: 35R11, 93B05, 30E05, 65M06.

\section{Introduction}

\subsection{Motivation}

In many realistic models coming from different branches of sciences (e.g. physics, chemistry, engineering), fractional Laplacians naturally arise to describe various phenomena.

More specifically, since the seminal paper by Richardson on turbulence diffusion [39], systems with weaker/strongly diffusivity (subdiffusion, superdiffusion) modelled by fractional equations are frequently used and are usually related with Brownian diffusion and the deviation of the statistics of fluctuation from the Gaussian law, see e.g. [14, 30, 43] and the references therein. Notably, examples of fractional parabolic dynamical system in the subdiffusive regime include (but

\footnotetext{
${ }^{*}$ CEREMADE, Université Paris-Dauphine \& CNRS UMR 7534, PSL Research University, 75016 Paris, France \& McTAO, Inria Sophia Antipolis, Université Côte d'Azur, CNRS, France. E-mail: lissy@ceremade.dauphine.fr.

${ }^{\dagger}$ Department of Mathematics, University of Craiova, 200585, Romania. E-mail: ionelroventa@yahoo.com.
} 
or not limited to): transport in amorphous semiconductors ([41]), nuclear magnetic resonance diffusometry in porous media $([22])$, transport on fractal geometries $([38])$, dynamics of a bead in a polymeric network $([1])$, etc.

Concerning hyperbolic equations or systems involving fractional Laplacian, they are often used in order to model attenuation (and then velocity dispersion) that characterizes the wave propagation in real media, notably viscoelastic ones. The first modelization using only fractional Laplacian (instead of fractional time derivatives) has been derived in [11]. This approach has been extended to many different cases notably in $[8,46]$, and for some heterogeneous media in [52]. Let us mention that some numerical studies of the viscoelastic wave equation with fractional Laplacian are notably proposed in $[10,44,51,47]$.

From the point of view of control theory, the study of models involving fractional Laplacians has been recently studied, see e.g. [33] for the internal controllability of the one-dimensional fractional heat and wave equations, [35] for a multi-dimensional result in the superdiffusive regime, [4] for internal controllability results in the multi-dimensional case for fractional Schrödinger or wave equations, [50] for an extension for measurable sets of control and and [27, 28, 29] for precise studies of the cost of controllability for one-dimensional fractional heat or Schrödinger equations.

Let us mention that many definitions of the fractional Laplacians co-exist (see e.g. [42]). However, they basically have the same behaviour at high frequency, meaning that choosing one definition instead of another should not change the controllability properties of the system under study, even if it may cause some additional technical difficulties. Here, for the sake of simplicity, we will consider the spectral definition of the fractional Laplace operator, which has the great advantage to enjoy explicit eigenfunctions and eigenvalues.

On the other hand, the approximation of controls of PDEs is a very popular direction of research and has attracted a lot of attention these last twenty years, due to the necessity of understanding how to achieve effective implementation of controls problems. This study has been initialized in [21] for the boundary controls of the wave equation and in [25] concerning the boundary controls beam equation (these equations appear to be limiting cases of the present study, in a sense explained in details afterwards). However, to our knowledge, the question of the approximation of controls in the case of fractional wave equations has never been investigated so far, despite its interest from a theoretical and applicative point of view. To conclude, let us mention that a numerical study of the one-dimensional fractional heat equation with finite element method has been performed in $[5]$.

The aim of the present paper is to study the semi-discrete approximation of the controls of a one-dimensional evolution problem of hyperbolic type involving a family of spectral fractional Laplacian. Note that a more realistic framework would be to consider a fully discretized (i.e. in space and time) version of the problem. However, in the case of conservative systems, the study of a semi-discrete version is sufficient to derive also a result for a fully discretized version of the system under study, involving some natural CFL-like condition on both time and space discretization steps (for more details see [15] and notably Section 4.2 of this article).

Mathematically speaking, our model can be seen as an intermediate case between the cases of the wave equation and the beam equation. We consider the simplest numerical approximation of such an equations as a finite difference semi-discrete scheme for the approximation of internal controls. It is well-known that, due to the high frequency numerical spurious oscillations, the uniform (with respect to the mesh-size) controllability property of the semi-discrete model fails in the natural 
setting. Consequently, the convergence of the approximate internal controls corresponding to an initial data in the energy space cannot be guaranteed.

Many possibilities have been proposed to overcome this difficulty: a Tychonov regularization of the HUM cost functional (see $[21,49]$ ), a change of the numerical scheme (mixed finite elements [9], vanishing viscosity $[6,32]$ and other type of finite difference schemes [37]), the introduction of non-uniform meshes $([18,19])$, an approximation of discrete controls $[12]$ (which does not lead exactly the discrete solution to zero, but converges to an exact control of the continuous problem), and finally an appropriate filtering technique, introduced in [31] and notably used in [13, 34, 26] in the context of wave or beam equation, which consists in relaxing the control requirement by controlling only the low-frequency part of the solution. This later approach will be considered in this paper.

Our approach consider a filtering technique which cut the frequencies of the initial conditions. Even if the initial condition is filtered, the control will excite all frequencies. This creates a lot of technical difficulties, because the spectral is not uniform with respect to the discretization step $h$ (see Remark 1.1). For more general uniform controllability results by using filtered spaces and resolvent estimates, the interested reader is referred to $[16,17,36]$.

Most of the above results treated the boundary controllability problems and we have seen that the negative results are due to the bad numerical approximation of the high eigenmodes. Since we are dealing with a fractional Laplacian problem of order $\alpha \in(1,2)$, and the question of prescribing non-homogeneous boundary conditions for these kinds of fractional Laplace operators is a tricky question (see e.g. [48]), we will not consider the case of boundary controls here, but rather the case when the control acts in the interior of the domain.

In the case $\alpha=2$ (i.e. the beam equation), the spurious numerical high eigenmodes do not play such an important role as in the case of boundary controls and the uniform controllability property holds automatically. For more details, see [6, Section 7].

The case $\alpha \in[1,2)$ is more intricate. The best result in the case $\alpha=1$ (i.e. the wave equation) has recently been obtained by the authors in [26] for boundary controls (the same result would hold for internal controls). It is notably proved an optimal result with respect to the range of filtration of the initial condition, together with precise (but non-optimal) estimates on the minimal time of uniform controllability. Even if the spirit of the present article is quite similar to [26], we would like to emphasize that the present study is more intricate and is far from being a direct consequence of the computations made in [26]. This is due to the following facts:

- Contrary to the corresponding product in [26], it is not clear at all that the product $R_{m}(x)$ is uniformly bounded with respect to the discretization step $h$ and $x$ in the all range $|x| \leqslant$ $(2 / h)^{\alpha}$. Notably, it can be proved numerically that the product $S_{m}$ is not bounded in the previous range. Hence, we had to understand what kind of estimates are needed, and to exhibit some very precise (and quite tricky to prove, see Section 2.2) estimates in this range, that depend both on $x, h$ and the discrete eigenvalues (see Remark 2.2), and are difficult to handle. These estimates are likely to be non-optimal but are enough for our purpose.

- The use of the Paley-Wiener Theorem requires to compensate the explosion of the product $R_{m}$ by an adequate multiplier. Because of the bad behaviour of the product $R_{m}$ emphasized above, the construction given in [26] is not sufficient to ensure the uniform boundedness of the discrete control with respect to $h$. Here, we use a new construction of the multiplier in the context of semi-discrete control problems. This construction is made of two parts. 
The first part is similar to the multiplier is similar to the one given in [26], and enables to compensate $R_{m}(x)$ for high $|x|$. The second part is similar to the construction used in [29] in the context of continuous fractional control problems, and is used to compensate the bad behaviour of the product $R_{m}(x)$ for low $|x|$ (this is the main difference with [26]). However, the use we make of this multiplier is quite different from the continuous case. Notably, we must analyze very precisely in different ranges how it can compensate the product $R_{m}$ by obtaining at the end uniform estimates in $h$ (see Section 2.3). To our knowledge, such a (quite complex) construction of a multiplier has never been considered in the context of semi-discrete problems, and might be of interest for a larger class of semi-discrete control problems. Note that for this later construction, it is crucial that $\alpha>1$.

- Contrary to [26] (and many papers on this topic), we are able to work in the natural energy space. This is due to the use of a refinement of the moment method that has been introduced in [24] and rediscovered recently in [7]. However, this construction requires to be much more careful about the estimation of the $L^{2}$-norm of the discrete control introduced in (3.1). It is also likely that the use of this refined moment method is of wider interest and might be used in many other context, probably improving many results of the literature concerning technical restrictions on the space of initial conditions.

Let us mention some perspectives and open question related to the present work. Concerning the model under study, one very natural question is to ask if we can study non-uniform mesh grids with the same technique. This will clearly require a deeper understanding of the phenomena under study, since the present work mainly relies on the fact that we know exactly the eigenvectors and eigenvalues of our discrete operator.

To finish, a more long-term remaining open question consists in finding the sharp filtering scale in a general setting, which should depend both on the defect of uniform discrete spectral gap, the asymptotics of the discrete/continuous eigenvalues and properties of localization of the discrete eigenvectors. We hope that the present study can be of help to reach this distant goal.

\subsection{Presentation of the problem}

This paper is concerned with the internal null-controllability (which is equivalent to the exact internal controllability) for a finite-difference semi-discrete scheme of the one-dimensional fractional wave equation on the space interval $(0,1)$.

More precisely, let us consider the 1-D Laplacian $\Delta$ with domain $H^{2}(0,1) \cap H_{0}^{1}(0,1)$, and state space $L^{2}(0,1)$. It is well-known that $-\Delta: D(\Delta) \rightarrow L^{2}(0,1)$ is a positive definite operator with compact resolvent, the $k$-th eigenvalue is $\lambda_{k}=k^{2} \pi^{2}$, with associated normalized eigenvector $e_{k}(x):=\sqrt{2} \sin (k \pi x)$.

Thanks to the continuous functional calculus for positive self-adjoint operators, one can define any positive power of $-\Delta$. We consider some $s>0$. Then, we introduce

$$
D\left(\left(-\Delta_{D}\right)^{s}\right)=\left\{f \in L^{2}(\Omega)\left|\sum_{k=1}^{\infty} \lambda_{k}^{2 s}\right|<f, e_{k}>\left.\right|_{L^{2}(0,1)} ^{2}<\infty\right\} .
$$


For $f=\sum_{k=1}^{\infty}<f, e_{k}>e_{k} \in D\left(\left(-\Delta_{D}\right)^{s}\right)$, we define

$$
\left(-\Delta_{D}\right)^{s} f(x)=\sum_{k=1}^{\infty} \lambda_{k}^{s}<f, e_{k}>e_{k} .
$$

Let us consider some $T>0$ and some $\alpha \in(1,2)$ (for $\alpha \geqslant 2$, the problem becomes trivial, see Remark 1.2). We consider $0<a<b<1$. We are interested in the following controlled equation:

$$
\begin{cases}u^{\prime \prime}(t, x)+\left(-\Delta_{D}\right)^{\alpha} u(t, x)=\chi_{(a, b)}(x) v(t, x) & t \in(0, T), x \in(0,1) \\ u(t, 0)=0 & t \in(0, T), \\ u(t, 1)=0 & t \in(0, T), \\ u(0, x)=u^{0}(x), \quad u^{\prime}(0, x)=u^{1}(x) & x \in(0,1)\end{cases}
$$

where $v \in L^{2}((0, T) \times(0,1))$ is a control, assumed to be localized in $(a, b)$. Let us remark that we have existence and uniqueness of solutions of (1.1) as soon as the initial condition $\left(u^{0}, u^{1}\right)$ lies in $D\left(\left(-\Delta_{D}\right)^{\alpha / 2}\right) \times L^{2}(0,1)$. More precisely, from now on we consider the following notations for the space of initial conditions

$$
\mathcal{H}=D\left(\left(-\Delta_{D}\right)^{\alpha / 2}\right) \times L^{2}(0,1)=\left\{\left(u^{0}, u^{1}\right)=\left.\sum_{k \in \mathbb{Z}^{*}} a_{k}\left(\frac{1}{k^{\alpha} i},-1\right) \sin (k \pi x)\left|\sum_{k \in \mathbb{Z}^{*}}\right| a_{k}\right|^{2}<\infty\right\} .
$$

It is well-known that for this kind of fractional wave equation (see [33, Section 6] for a result with a scalar control and an imposed profile, but the result can be easily extended to (1.1) using a technique similar to [24]), null-controllability for initial conditions in the space $\mathcal{H}$ holds in arbitrary small time if and only if $\alpha>1$.

Let $N \in \mathbb{N}^{*}$ and $h=\frac{1}{N+1}$. We introduce

$$
\begin{gathered}
U(t):=\left(\begin{array}{c}
u(t, h) \\
u(t, 2 h) \\
\ldots \\
u(t, N h)
\end{array}\right), \\
U^{0}:=\left(\begin{array}{c}
u^{0}(h) \\
u^{0}(2 h) \\
\ldots \\
u^{0}(N h)
\end{array}\right) \text { and } U^{1}:=\left(\begin{array}{c}
u^{1}(h) \\
u^{1}(2 h) \\
\ldots \\
u^{1}(N h)
\end{array}\right) .
\end{gathered}
$$

For any $T>0$, we consider the following semi-discrete space approximation of the fractional wave equation (1.1) by the following explicit finite-difference method, that we write directly in matrix form:

$$
\left\{\begin{array}{l}
U^{\prime \prime}+\mathcal{A}_{h}^{\alpha} U=\mathcal{B}_{h}\left(v_{h}(t)\right) \quad 1 \leq j \leq N, t \in(0, T), \\
U(0, \cdot)=U^{0}, \quad U^{\prime}(0, \cdot)=U^{1},
\end{array}\right.
$$


where $\mathcal{A}_{h} \in \mathcal{M}_{N}(\mathbb{R})$ is the discrete Laplacian given by

$$
\mathcal{A}_{h}:=\frac{1}{h^{2}}\left(\begin{array}{ccccccc}
2 & -1 & 0 & \ldots & \ldots & \ldots & 0 \\
-1 & 2 & -1 & 0 & \ldots & \ldots & 0 \\
0 & -1 & 2 & -1 & 0 & \ldots & 0 \\
\ldots & \ldots & \ldots & \ldots & \ldots & \ldots & \ldots \\
\ldots & \ldots & \ldots & \ldots & \ldots & \ldots & \ldots \\
0 & \ldots & 0 & 0 & -1 & 2 & -1 \\
0 & \ldots & \ldots & \ldots & 0 & -1 & 2
\end{array}\right)
$$

and $\mathcal{B}_{h} \in \mathcal{M}_{N}(\mathbb{R})$ is the discrete control operator given by

$$
\mathcal{B}_{h}\left(v_{1}, \ldots v_{N}\right):=\left(\chi_{(a, b)}(j h) v(j)\right)_{1 \leqslant j \leqslant N}^{T} .
$$

Note that $\mathcal{A}_{h}$ is a symmetric positive matrix, so that by usual continuous functional calculus, it is very easy to define any of its positive powers. Moreover, since the eigenfunctions and eigenvalues of $\mathcal{A}_{h}$ are known explicitly, it is also very easy to compute numerically such powers. To conclude, it is easy to prove that for any $\alpha \in(1,2)$, the semi-discrete scheme given by (1.3) is consistant of order (at least) 2 with respect to equation (1.1) (see [45, Theorem 1]), so that it can be proved to be convergent and hence to be a reasonable approximation of (1.1).

Given $T>0, h>0$ and $\left(\left(u_{j}^{0}, u_{j}^{1}\right)\right)_{1 \leq j \leq N} \in \mathbb{C}^{2 N}$, we study the existence of a control function $v_{h} \in L^{2}(0, T)$ such that the solution of the equation (1.3) verifies

$$
u_{j}(T)=u_{j}^{\prime}(T)=0 \quad(j=1,2, \ldots, N) .
$$

More precisely, our aim is to study the existence of a uniformly bounded sequence of controls $\left(v_{h}\right)_{h>0}$ with respect to the mesh size $h$, by using the moment method introduced in [20] for the boundary controllability of the heat equation. Note that here, contrary to what is done in general, the control is not a scalar control (i.e. it is not of the form $f(x) g(t)$, with $f$ some imposed profile in space in an adequate class, and $g \in L^{2}(0, T)$ some scalar control function). For the continuous system (1.1), the control lives in an infinite dimensional subspace, whereas in the discrete case of (1.3), the control lives in a finite dimensional space of dimension $N$. This generalization of the moment method has been introduced for the internal control of the wave equation in [24], used notably in the context of parabolic equations in $[3,7]$ and studied in a more general setting in [2]. It enables us to get rid of technical assumptions on the initial conditions, that can be taken in the natural energy space $\mathcal{H}$, contrary to many previous results in the literature.

On the other hand, the choice of an appropriate approximation $\left(\left(u_{j}^{0}, u_{j}^{1}\right)\right)_{1 \leq j \leq N} \in \mathbb{C}^{2 N}$ for the initial datum $\left(u^{0}, u^{1}\right) \in \mathcal{H}$ is very important in order to ensure the uniform boundedness of the sequence of controls $\left(v_{h}\right)_{h>0}$.

Let us introduce some additional notations. We consider $\mathcal{L}_{h}^{\alpha} \in \mathcal{M}_{2 N \times 2 N}(\mathbb{R})$ given by

$$
\mathcal{L}_{h}^{\alpha}:=\left(\begin{array}{cc}
0 & -I_{n} \\
\mathcal{A}_{h}^{\alpha} & 0
\end{array}\right),
$$

where $I_{n} \in \mathcal{M}_{n}(\mathbb{R})$ is the identity matrix of size $n$. 
The eigenvalues of the discretization matrix $\mathcal{L}_{h}^{\alpha}$ are given by the family $\left(i \lambda_{n}(h)\right)_{1 \leq|n| \leq N}$, where

$$
\lambda_{n}(h)=\operatorname{sgn}(n)\left(\frac{2}{h} \sin \left(\frac{|n| \pi h}{2}\right)\right)^{\alpha}, \quad 1 \leq|n| \leq N,
$$

and the corresponding eigenvectors are

$$
\Phi_{h}^{n}=\left(\begin{array}{c}
\frac{1}{i \lambda_{n}} \varphi_{h}^{n} \\
-\varphi_{h}^{n}
\end{array}\right) \quad(1 \leq|n| \leq N)
$$

where

$$
\left(\varphi_{h}^{n}\right)_{1 \leq|n| \leq N}=\left(\begin{array}{c}
\sin (n \pi h) \\
\sin (2 n \pi h) \\
\cdots \\
\sin (N n \pi h)
\end{array}\right) \in \mathbb{C}^{N}
$$

For the sake of simplicity, we will often write $\lambda_{n}$ instead of $\lambda_{n}(h)$ in what follows, when there is no ambiguity. Note that the eigenvalues of the matrix $\mathcal{A}_{h}^{\alpha}$ are $\left(\lambda_{n}^{2}\right)_{1 \leq n \leq N}$ and $\left(\Phi_{h}^{n}\right)_{1 \leq|n| \leq N}$ forms an orthonormal basis in $\mathbb{C}^{2 N}$ (see [31, Proposition 3.1]).

We conclude this preliminary section with some gap estimates which we believe that determine the real behavior of the sequence of discrete controls.

Remark 1.1. Let us consider $\alpha \geq 1$ and $M>0$. We remark that the gap between the eigenvalues $\lambda_{n}(h)$ from (1.4) depends dramatically on the position of $\alpha$. More precisely, it is easy to prove that, for any $h>0$ sufficiently small, the following statements hold:

- if $\alpha=1$ then

$$
\left|\lambda_{n+1}-\lambda_{n}\right|= \begin{cases}\Theta(1) & 1 \leq|n| \leq \delta N, \quad \forall \delta \in(0,1) \\ \Theta(h) & N-M \leq|n| \leq N\end{cases}
$$

- if $\alpha \in(1,2)$ then

$$
\left|\lambda_{n+1}-\lambda_{n}\right|= \begin{cases}\Theta(1) & 1 \leq|n| \leq M \\ \Theta\left(\frac{1}{h^{\alpha-1}}\right) & \frac{\delta N}{2} \leq|n| \leq \delta N, \quad \forall \delta \in(0,1) \\ \Theta\left(h^{2-\alpha}\right) & N-M \leq|n| \leq N\end{cases}
$$

- if $\alpha=2$ then

$$
\left|\lambda_{n+1}-\lambda_{n}\right|=\left\{\begin{array}{l}
\Theta(1) \quad 1 \leq|n| \leq M \\
\Theta\left(\frac{1}{h}\right) \quad \frac{\delta N}{2} \leq|n| \leq \delta N, \quad \forall \delta \in(0,1) \\
\Theta(1) \quad N-M \leq|n| \leq N
\end{array}\right.
$$

- if $\alpha>2$ then

$$
\left|\lambda_{n+1}-\lambda_{n}\right|= \begin{cases}\Theta(1) & 1 \leq|n| \leq M \\ \Theta\left(\frac{1}{h^{\alpha-1}}\right) & \frac{\delta N}{2} \leq|n| \leq \delta N, \quad \forall \delta \in(0,1) \\ \Theta\left(\frac{1}{h^{\alpha-2}}\right) & N-M \leq|n| \leq N\end{cases}
$$

where the Bachmann-Landau notation Big Theta has to be understood in the asymptotics $h \rightarrow 0$. 
Remark 1.2. Note that, our problem deals with the difficult case $\alpha \in(1,2)$. Indeed, if $\alpha \geqslant 2$, we proceed as in [6, Section 7](see also[25]), taking into account the uniform gap estimates given in Remark 1.1, we can use a generalization of Ingham's inequality [[23], Theorem 4.6].

More precisely, if we have a family with the gap tending to infinity when $h$ goes to zero and another finite family (depending on $T$ ) with gap $O(1)$, then we can use an Ingham-type inequality for the reunion of the two families, for any time $T>0$ (with a constant depending only on $T$ ).

Hence, for $\alpha \geq 2$ the uniform controllability property holds automatically in the context of internal controls.

\subsection{Main results and structure of the article}

Let us consider some increasing function $f: \mathbb{N}^{*}: \rightarrow \mathbb{N}^{*}$ (that will play the role of our filtration). Our main Theorem is the following.

Theorem 1.1. Let

$$
\left(u_{0}, u_{1}\right)=\sum_{k \in \mathbb{Z}^{*}} a_{k}\left(\frac{1}{k^{\alpha} i},-1\right) \sin (k \pi x) \in \mathcal{H}
$$

Assume that

$$
\lim _{N \rightarrow \infty} f(N)=\infty \text { and } \Gamma:=\limsup _{N \rightarrow \infty} \frac{f(N)}{N}<1 .
$$

For any $\left(u_{j}^{0}, u_{j}^{1}\right)_{1 \leq j \leq N}=\sum_{1 \leq|n| \leq f(N)} a_{n} \Phi_{h}^{n}$ there exists a uniformly bounded sequence of controls $\left(v_{h}\right)_{h>0}$ for the discrete control problem (1.3) verifying that for any $h>0$, the solution $\left(u_{1}, \ldots u_{N}\right)$ of (1.3) verifies $\left(u_{1}(T), \ldots, u_{N}(T)\right)=(0, \ldots, 0)$.

Remark that our theorem is optimal in the sense that we can work on the natural energy space $\mathcal{H}$ and that uniform controllability may fail if we do not filtrate the initial conditions. This last phenomenon has notably been highlighted in [31] in the case of the wave equation $\alpha=1$, and is stated in the following Theorem.

Theorem 1.2. Let

$$
\left(u_{0}, u_{1}\right)=\sum_{k \in \mathbb{Z}^{*}} a_{k}\left(\frac{1}{k^{\alpha} i},-1\right) \sin (k \pi x) \in \mathcal{H}
$$

We introduce $\left(u_{j}^{0}, u_{j}^{1}\right)_{1 \leq j \leq N}=\sum_{1 \leq|n| \leq N} a_{n} \Phi_{h}^{n}$. There exists initial conditions $\left(u_{0}, u_{1}\right)$ for which no sequence of controls $\left(v_{h}\right)_{h>0}$ for the discrete control problem (1.3) (i.e., verifying that for any $h>0$, the solution $\left(u_{1}, \ldots u_{N}\right)$ of $(1.3)$ verifies $\left.\left(u_{1}(T), \ldots, u_{N}(T)\right)=(0, \ldots, 0)\right)$ can be uniformly bounded in $h$.

Theorem 1.2 is standard and relies on the non-uniform gap property at high frequency stated in Remark 1.1. For the sake of completeness, Theorem 1.2 is proved in Appendix A.

As a corollary of Theorem 1.1, we obtain the following convergence result. 
Corollary 1. Let $\left(u^{0}, u^{1}\right)=\sum_{1 \leq|n| \leq f(N)} a_{n} \Phi^{n}$ be the initial datum for the continuous problem (1.1) and let us consider the initial datum of the semi-discrete problem (1.3)

$$
\left(u_{j}^{0}, u_{j}^{1}\right)_{1 \leq j \leq N}=\sum_{1 \leq|n| \leq f(N)} a_{n} \tilde{\Phi}^{n}(h)
$$

where $\tilde{\Phi}^{n}(h)$ is the discretization of the eigenfunction $\Phi^{n}$ of the form

$$
\tilde{\Phi}^{n}(h)=\left(\left(\frac{1}{n^{\alpha} i} \sin (j n \pi h)\right)_{1 \leq j \leq N},(-\sin (j n \pi h))_{1 \leq j \leq N}\right)^{T} .
$$

Let $v \in L^{2}(0, T)$ a weak limit of a subsequence of the bounded controls $\left(v_{h}\right)_{h>0}$ given by Theorem 1.1, with initial datum given in (1.6). Then $v$ is a control for the continuous problem (1.1).

The structure of the article is as follows.

In Section 2.1, we will give some spectral properties of the semi-discretized problem and we will set our equivalent moment problem, that we will solve by applying the Paley-Wiener Theorem. This means that one has first to study some appropriate product (the product $R_{m}$ introduced in (2.26)) involving the discrete eigenfunctions and then construct an adequate multiplier. As already mentioned, contrarily to many work on the controllability of distributed systems, we do not impose a fixed profile in space and we do not work with a scalar control depending only on time. This enables to get rid of technical assumptions on the initial condition (see e.g. [33, Section $6]$ ) and to work in the natural energy space, which is also a novelty of this article.

In Section 2.2, we will give crucial estimates on an auxiliary product $P_{m}$, defined in (2.8) and related to $R_{m}$ by (2.27). The main novelty is that we are able to prove precise (and difficult) estimates on this product, that is bounded independently on the parameter $h$ in some appropriate range (see (2.9)). This leads to a new and very precise estimate on the product $R_{m}$.

It is worth to mention that, in contrast to the case of wave equation $\alpha=1$, where the Weierstrass product $R_{m}$ is bounded in the range less than $\frac{2}{h}$, for $\alpha \in(1,2)$ we have a smaller domain on boundedness. This fact is really crucial for the wave case and we need to solve this problem with a precise and smart multiplier.

Concerning the multiplier, in Section 2.3, we will use a new construction in the context of semidiscrete problems, taking into account both regimes described above. We will mix a part similar to the construction of [26] for the semi-discrete wave equations in order to compensate the "bad" dependance in $h$ of $R_{m}$ for large $x$, and another part, which is similar to the construction made in [29] in the case of (continuous) fractional heat or Schrödinger equations, and will be useful to compensate the estimation of $R_{m}$ for small $x$ coming from inequality (2.9).

In Section 2.4, we construct our biorthogonal family using the Paley-Wiener Theorem, and we give some estimations on this biorthogonal family.

Finally, Sections 3.1 and 3.2 are devoted to the proofs of Theorem 1.1 and Corollary 1, i.e. the construction of the discrete controls, the proof of uniform estimates with respect to $h$ for filtered initial conditions, and the proof of convergence result for the discretized control. 


\section{The moment problem. Construction of a biorthogonal family}

\subsection{Reduction to a moment problem}

The aim of this subsection is to explain how the control result given in Theorem 1.1 can be reduced to the study of an adequate moment problem.

We start the study of the controllability properties for (1.3) by considering the following adjoint system:

$$
\begin{cases}w_{j}^{\prime \prime}(t)+\left(\mathcal{A}_{h}^{\alpha} W\right)_{j}=0 & 1 \leq j \leq N, t>0, \\ w_{0}(t)=0 & t \in(0, T), \\ w_{N+1}(t)=0 & t \in(0, T) \\ w_{j}(0)=w_{j}^{0}, \quad w_{j}^{\prime}(0)=w_{j}^{1} & 1 \leq j \leq N\end{cases}
$$

where $W(t)=\left(w_{1}(t), \ldots, w_{N}(t)\right)^{T}$.

We can rewrite the adjoint system (2.1) in an abstract Cauchy form as

$$
\left\{\begin{array}{l}
W^{\prime \prime}(t)+\mathcal{A}_{h}^{\alpha} W=0, t>0 \\
W(0)=W^{0}, \quad W^{\prime}(0)=W^{1}
\end{array}\right.
$$

where $W(t)=\left(w_{1}(t), \ldots, w_{N}(t)\right)^{T}, W^{0}=\left(w_{j}^{0}\right)_{1 \leq j \leq N}$ and $W^{1}=\left(w_{j}^{1}\right)_{1 \leq j \leq N}$.

Moreover, if we consider $Z=\left(W, W^{\prime}\right)^{T}$, we have the following equivalent form

$$
\left\{\begin{array}{l}
Z^{\prime}(t)+L_{h}^{\alpha} Z(t)=0, t>0 \\
Z(0)=Z^{0}=\left(W^{0}, W^{1}\right)^{T}
\end{array}\right.
$$

where $\mathcal{L}_{h} \in \mathcal{M}_{2 N \times 2 N}(\mathbb{R})$ is given by

$$
L_{h}^{\alpha}:=\left(\begin{array}{cc}
0 & -I \\
\mathcal{A}_{h}^{\alpha} & 0
\end{array}\right)
$$

Let us define the corresponding discrete energy as

$$
E(t)=\frac{h}{2} \sum_{j=0}^{N}\left(\left(\mathcal{A}_{h}^{\alpha} W\right)_{j} w_{j}+\left|w_{j}^{\prime}(t)\right|^{2}\right) .
$$

In fact, the above energy is generated by the following inner product

$$
\left\langle\left(\begin{array}{c}
W_{1} \\
U_{1}
\end{array}\right),\left(\begin{array}{c}
W_{2} \\
U_{2}
\end{array}\right)\right\rangle_{\alpha}=\left\langle\mathcal{A}_{h}^{\alpha} W_{1}, W_{2}\right\rangle+\left\langle U_{1}, U_{2}\right\rangle .
$$


We can easily deduce that, if $Z^{0}=\left(W^{0}, W^{1}\right)$ verifies

$$
Z^{0}=\sum_{1 \leq|n| \leq N} a_{n}^{0} \Phi_{h}^{n}
$$

then the solution of the system (2.2) is given by

$$
Z(t)=\sum_{1 \leq|n| \leq N} a_{n}^{0} e^{i \lambda_{n} t} \Phi_{h}^{n}
$$

Now, we have all the ingredients to derive our moment problem. Let us go back to equation (1.3).

The following lemma gives a variational characterization of the controllability problem (1.3).

Lemma 2.1. The problem (1.3) is controllable if and only if, for any initial datum $Z^{0}=$ $\left(u_{j}^{0}, u_{j}^{1}\right)_{1 \leq j \leq N} \in \mathbb{C}^{2 N}$ there exist a function $v_{h} \in\left(L^{2}(0, T)\right)^{N}$ such that

$$
\sum_{1 \leq j \leq N}\left(u_{j}^{0} \bar{w}_{j}^{1}-u_{j}^{1} \bar{w}_{j}^{0}\right)=\sum_{1 \leq j \leq N} \int_{0}^{T} \chi_{(a, b)}(j h) \bar{w}_{j}(t) v_{h}(t, j h) d t
$$

for any $\left(w_{j}^{0}, w_{j}^{1}\right)_{1 \leq j \leq N} \in \mathbb{C}^{2 N}$ and $w=\left(w_{1}(t), \ldots, w_{N}(t)\right)$ solution of the adjoint system (2.1).

Proof of Lemma 2.1 Let $\left(u_{j}^{0}, u_{j}^{1}\right)_{1 \leq j \leq N} \in \mathbb{C}^{2 N}$ and let $U=\left(u_{1}(t), \ldots, u_{N}(t)\right)$ be the corresponding solution of the primal system (1.3). We consider $\left(w_{j}^{0}, w_{j}^{1}\right)_{1 \leq j \leq N} \in \mathbb{C}^{2 N}$ and let $w=\left(w_{1}(t), \ldots, w_{N}(t)\right)$ be the corresponding solution of the adjoint system (2.1).

For each, $1 \leq j \leq N$, multiplying the $(j+N)$-th equation in (1.3) by $\bar{w}_{j}(t)$, integrating by parts and summing up we obtain

$$
\sum_{1 \leq j \leq N} \int_{0}^{T}\left(u_{j}^{\prime \prime}(t) \bar{w}_{j}(t)+\left(\mathcal{A}_{h}^{\alpha} U\right)_{j} \bar{w}_{j}(t)\right) d t=\sum_{1 \leq j \leq N} \int_{0}^{T} \chi_{(a, b)}(j h) \bar{w}_{j}(t) v_{h}(t, j h) d t,
$$

from where it follows

$$
\begin{gathered}
\left.\sum_{1 \leq j \leq N}\left(u_{j}^{\prime}(t) \bar{w}_{j}(t)-u_{j}(t) \bar{w}_{j}^{\prime}(t)\right)\right|_{0} ^{T} \\
+\sum_{1 \leq j \leq N} \int_{0}^{T} u_{j}(t)\left(\bar{w}_{j}^{\prime \prime}(t)+\left(\mathcal{A}_{h}^{\alpha} \bar{W}\right)_{j}\right) d t=\sum_{1 \leq j \leq N} \int_{0}^{T} \chi_{(a, b)}(j h) \bar{w}_{j}(t) v_{h}(t, j h) d t .
\end{gathered}
$$

Finally, since the controllability of problem (1.3) is equivalent with

$$
u_{j}(T)=u_{j}^{\prime}(T)=0 \quad(j=1,2, \ldots, N),
$$

the proof of Lemma 2.1 is complete.

Remark 2.1. Note that, using similar estimates as in [31, Remark 6], the left hand term in (2.5) for $\left(u_{j}^{0}, u_{j}^{1}\right)_{1 \leq j \leq N}=\Phi_{h}^{k}$ and $\left(w_{j}^{0}, w_{j}^{1}\right)_{1 \leq j \leq N}=\Phi_{h}^{n}$ is given by

$$
\sum_{1 \leq j \leq N}\left(u_{j}^{0} \bar{w}_{j}^{1}-u_{j}^{1} \bar{w}_{j}^{0}\right)=\frac{i}{h \lambda_{n}} \delta_{n k} .
$$


Now, we are able to transform our controllability problem into a moment problem.

Proposition 2.1. The problem (1.3) is controllable if and only if, for any initial datum $Z^{0}=$ $\sum_{1 \leq|k| \leq N} \alpha_{k} \Phi_{h}^{k} \in \mathbb{C}^{2 N}$ there exist a function $v_{h} \in\left(L^{2}(0, T)\right)^{N}$ such that

$$
h \sum_{1 \leq j \leq N} \chi_{(a, b)}(j h) \sin (j \pi n h) \int_{0}^{T} e^{-i \lambda_{n} t} v_{h}(t, j h) d t=\alpha_{n} \quad(1 \leq|n| \leq N) .
$$

Proof of Proposition 2.1 Using Lemma 2.1, we deduce that system (1.3) is controllable if and only if, for any initial datum $\left(u_{j}^{0}, u_{j}^{1}\right)_{1 \leq j \leq N}$, there exists a function $v_{h} \in L^{2}(0, T)$ such that $(2.5)$ holds for

$$
\left(w_{j}^{0}, w_{j}^{1}\right)_{1 \leq j \leq N}=\Phi_{h}^{n} \quad(1 \leq|n| \leq N),
$$

which gives the solution from $(2.4)$ as

$$
Z(t)=e^{i \lambda_{n} t} \Phi_{h}^{n}
$$

Hence, for $\left(u_{j}^{0}, u_{j}^{1}\right)_{1 \leq j \leq N}=\sum_{1 \leq|k| \leq N} \alpha_{k} \Phi_{h}^{k} \in \mathbb{C}^{2 N}$ from (2.6) we have

$$
\sum_{1 \leq j \leq N}\left(u_{j}^{0} \bar{w}_{j}^{1}-u_{j}^{1} \bar{w}_{j}^{0}\right)=\frac{i \alpha_{n}}{h \lambda_{n}} .
$$

Since $\bar{w}_{j}(t)=-\frac{\sin (j \pi n h)}{i \lambda_{n}} e^{-i \lambda_{n} t}$ we get

$$
h \sum_{1 \leq j \leq N} \chi_{(a, b)}(j h) \sin (j \pi n h) \int_{0}^{T} e^{-i \lambda_{n} t} v_{h}(t, j h) d t=\alpha_{n},
$$

hence the proof of (2.7) is finished.

From now on, our aim is to construct and evaluate an explicit biorthogonal sequence to the family $\left(e^{i \lambda_{n} t}\right)_{1 \leq|n| \leq N}$ in $L^{2}\left(-\frac{T}{2}, \frac{T}{2}\right)$.

\subsection{New product estimates}

In this subsection we introduce a product $R_{m}$ which has the property that $R_{m}\left(\lambda_{n}\right)=\delta_{m n}$. We are able to get very precise estimates of the product $R_{m}$ on the real axis.

For every $1 \leq|m| \leq f(N)$, we define first the following auxiliary product, that will be of interest for what follows (see notably $(2.27)$ ):

$$
P_{m}(x):=\prod_{\substack{1 \leq n \leq N \\ n \neq m}}\left(1-\frac{x}{\lambda_{n}^{2}-\lambda_{m}^{2}}\right) .
$$

We have the following result. 
Proposition 2.2. Let $A>0$. There exists a constant $D_{A}>0$ (depending on $\alpha>1$ and $A$ ) such that for every $h>0$ small enough, $|m| \leqslant f(N)$ and every $x$ such that $|x| \leqslant \frac{A}{h^{2 \alpha}}$, we have

$$
\left|P_{m}(x)\right| \leqslant D_{A} \exp \left(D_{A}|x|^{\frac{1}{2 \alpha}}\right) .
$$

Moreover, we also have the existence of $C>0$ (depending only on $\alpha$ and $\Gamma$ defined in (1.5)) such that for any $x \in \mathbb{R}$,

$$
\left|P_{m}(x)\right| \leqslant C \exp \left(C\left(h|x|^{\frac{1}{\alpha}}+\frac{|x| \ln (m)}{m^{2 \alpha-1}}\right)\right)
$$

Proof of Proposition 2.2. In all what follows, $C>0$ is a constant depending only on $\alpha$ and $\Gamma$ (and possibly $A$ if we restrict to the range $|x| \leqslant \frac{A}{h^{2 \alpha}}$ ) that may vary from inequality to inequality. We assume without loss of generality that $\lambda_{m} \geqslant 0$ (the case $\lambda_{m} \leqslant 0$ can be treated in a similar way). First of all, we remark that

$$
\left|P_{m}(x)\right|=\prod_{\substack{1 \leq n \leq N \\ n \neq m}}\left|1-\frac{x}{\lambda_{n}^{2}-\lambda_{m}^{2}}\right| \leqslant \prod_{\substack{1 \leq n \leq N \\ n \neq m}}\left(1+\frac{|x|}{\left|\lambda_{n}^{2}-\lambda_{m}^{2}\right|}\right)=Q_{m}^{1}(x) Q_{m}^{2}(x),
$$

where

$$
Q_{m}^{1}(x)=\prod_{\substack{1 \leq n \leq N \\ n \neq m, n+m \geqslant \frac{1}{h}}}\left(1+\frac{|x|}{\left|\lambda_{n}^{2}-\lambda_{m}^{2}\right|}\right), \quad Q_{m}^{2}(x)=\prod_{\substack{1 \leq n \leq N \\ n \neq m, n+m<\frac{1}{h}}}\left(1+\frac{|x|}{\left|\lambda_{n}^{2}-\lambda_{m}^{2}\right|}\right) .
$$

We have that

$$
\lambda_{n}^{2}-\lambda_{m}^{2}=\left(\frac{2}{h}\right)^{2 \alpha}\left(\sin \left(\frac{n \pi h}{2}\right)^{2 \alpha}-\sin \left(\frac{m \pi h}{2}\right)^{2 \alpha}\right) .
$$

- First regime: $n+m \geqslant \frac{1}{h}$.

Since $\left|x^{\alpha}-y^{\alpha}\right| \geq|x-y|^{\alpha}$, for all $x, y \in \mathbb{R}$ and $\alpha>1$, it follows that

$$
\left|\lambda_{n}^{2}-\lambda_{m}^{2}\right| \geqslant\left(\frac{2}{h}\right)^{2 \alpha}\left|\sin \left(\frac{n \pi h}{2}\right)^{2}-\sin \left(\frac{m \pi h}{2}\right)^{2}\right|^{\alpha}
$$

Following the computations made in [26] and using the inequality $\sin \left(\frac{\pi x}{2}\right) \geqslant x$, true for any $x \in[0,1]$, we deduce that

$$
\left|\lambda_{n}^{2}-\lambda_{m}^{2}\right| \geqslant\left(\frac{2}{h}\right)^{2 \alpha}\left|\sin \left(\frac{(n-m) \pi h}{2}\right) \sin \left(\frac{(n+m) \pi h}{2}\right)\right|^{\alpha} \geqslant\left(\frac{4|n-m|}{h}\right)^{\alpha}\left|\sin \left(\frac{(n+m) \pi h}{2}\right)\right|^{\alpha} .
$$

Now, let us study into more details $\sin \left(\frac{(n+m) \pi h}{2}\right)$. Using our hypothesis $n+m \geqslant \frac{1}{h}$, we have that $\frac{h(n+m) \pi}{2} \geqslant \frac{\pi}{2}$. Since $x \mapsto \sin (x)$ is decreasing on $[\pi / 2, \pi]$, we deduce that

$$
\sin \left(\frac{(n+m) \pi h}{2}\right) \geqslant \sin \left(\frac{(N+f(N)) \pi h}{2}\right) \geqslant \sin \left(\frac{N}{N+1}\left(1+\frac{f(N)}{N}\right) \frac{\pi}{2}\right) .
$$


Using Hypothesis (1.5), we obtain that $\lim \sup _{h \rightarrow 0} \frac{N}{N+1}\left(1+\frac{f(N)}{N}\right) \frac{\pi}{2}=(1+\Gamma) \frac{\pi}{2}$. Hence, there exists $C<1$ such that for $h$ small enough, we have

$$
\sin \left(\frac{N}{N+1}\left(1+\frac{f(N)}{N}\right) \frac{\pi}{2}\right) \geqslant \sin \left((1+C) \frac{\pi}{2}\right) .
$$

Since $C<1$, we deduce that $\sin \left((1+C) \frac{\pi}{2}\right)>C>0$, so that finally, we obtain

$$
\sin \left(\frac{(n+m) \pi h}{2}\right) \geqslant C \text {. }
$$

Hence, we deduce

$$
\left|\lambda_{n}^{2}-\lambda_{m}^{2}\right| \geqslant C\left(\frac{|n-m|}{h}\right)^{\alpha}
$$

We deduce by plugging this inequality into the first term of (2.12) that

$$
Q_{m}^{1}(x) \leqslant \prod_{\substack{1 \leq n \leq N \\ n \neq m}}\left(1+\frac{C h^{\alpha}|x|}{|n-m|^{\alpha}}\right) .
$$

Then,

$$
\begin{aligned}
\ln \left(Q_{m}^{1}(x)\right) & \leqslant \sum_{\substack{1 \leq n \leq N \\
n \neq m}} \ln \left(1+\frac{C h^{\alpha}|x|}{|n-m|^{\alpha}}\right) . \\
& \leqslant \sum_{\substack{1 \leq n \leq N \\
n \neq m}} \int_{0}^{C|x| h^{\alpha}} \frac{d t}{t+|n-m|^{\alpha}} \\
& \leqslant \int_{0}^{C|x| h^{\alpha}} \sum_{\substack{1 \leq n \leq N \\
n \neq m}} \frac{d t}{t+|n-m|^{\alpha}} \\
& \leqslant \int_{0}^{C|x| h^{\alpha}} \sum_{\substack{1 \leq n \leq N \\
n \neq m}}^{\infty} \int_{|n-m|^{\alpha}}^{\infty} \frac{d s}{(t+s)^{2}} d t \\
& \leqslant \int_{0}^{C|x| h^{\alpha}} \int_{\substack{\left|N-m f^{\alpha} \\
\inf _{n}\right| n-\left.m\right|^{\alpha}}} \frac{L_{m}(s) d s}{(t+s)^{2}} d t
\end{aligned}
$$

where

$$
L_{m}(s):=\operatorname{card}\left\{1 \leq n \leqslant N \text { s.t. }|n-m|^{\alpha} \leqslant s\right\} .
$$

It is easy to estimate this counting function. We have

$$
|n-m|^{\alpha} \leqslant s \Leftrightarrow\left(m-s^{\frac{1}{\alpha}}\right)^{+} \leqslant n \leqslant m+s^{\frac{1}{\alpha}} .
$$

Hence, we deduce that

$$
\begin{aligned}
\ln \left(Q_{m}^{1}(x)\right) & \leqslant \int_{0}^{C|x| h^{\alpha}} \int_{1}^{(N-m)^{\alpha}} \frac{\left(m+s^{\frac{1}{\alpha}}\right)-\left(m-s^{\frac{1}{\alpha}}\right)^{+}}{(t+s)^{2}} d s d t \\
& \leqslant C|x| h^{\alpha} \int_{1}^{(N-m)^{\alpha}} \frac{\left(m+s^{\frac{1}{\alpha}}\right)-\left(m-s^{\frac{1}{\alpha}}\right)^{+}}{s\left(s+C|x| h^{\alpha}\right)} d s
\end{aligned}
$$


We introduce the change of variables $s=m^{\alpha} t$. We deduce that

$$
\begin{aligned}
\ln \left(Q_{m}^{1}(x)\right) & \leqslant \frac{C|x| h^{\alpha}}{m^{\alpha-1}} \int_{\frac{1}{m^{\alpha}}}^{\frac{|N-m|^{\alpha}}{\left|m^{\alpha}\right|}} \frac{\left(1+t^{\frac{1}{\alpha}}\right)-\left(1-t^{\frac{1}{\alpha}}\right)^{+}}{t\left(t+\frac{C|x| h^{\alpha}}{m^{\alpha}}\right)} d t \\
& \leqslant \frac{C|x| h^{\alpha}}{m^{\alpha-1}} \int_{0}^{\infty} \frac{\left(1+t^{\frac{1}{\alpha}}\right)-\left(1-t^{\frac{1}{\alpha}}\right)^{+}}{t\left(t+\frac{C|x| h^{\alpha}}{m^{\alpha}}\right)} d t .
\end{aligned}
$$

Let us introduce, for $y \geqslant 0$,

$$
U(y)=\int_{0}^{1} \frac{2 t^{\frac{1}{\alpha}}}{t(t+y)} d t \text { and } V(y)=\int_{1}^{\infty} \frac{1+t^{\frac{1}{\alpha}}}{t(t+y)} d t .
$$

Using the change of variables $t=y v^{\alpha}$, we obtain

$$
U(y)=\int_{0}^{y^{-\frac{1}{\alpha}}} \frac{2 \alpha y v^{\alpha-1} y^{\frac{1}{\alpha}} v}{y^{2} v^{\alpha}\left(1+v^{\alpha}\right)} d v \leqslant y^{\frac{1}{\alpha}-1} \int_{0}^{\infty} \frac{2 \alpha}{\left(1+v^{\alpha}\right)} d v \leqslant C y^{\frac{1}{\alpha}-1} .
$$

Hence, we have obtained

$$
U(y) \leqslant \frac{C}{y^{1-\frac{1}{\alpha}}}
$$

On the other hand,

$$
V(y)=\int_{1}^{\infty} \frac{t^{\frac{1}{\alpha}}}{t(t+y)} d t+\int_{1}^{\infty} \frac{1}{t(t+y)} d t
$$

Concerning the first integral, using as before the change of variables $t=y v^{\alpha}$, we obtain that

$$
\int_{1}^{\infty} \frac{t^{\frac{1}{\alpha}}}{t(t+y)} d t \leqslant y^{1-\frac{1}{\alpha}} \int_{0}^{\infty} \frac{\alpha}{\left(1+v^{\alpha}\right)} d t \leqslant \frac{C}{y^{1-\frac{1}{\alpha}}}
$$

Concerning the second integral, its value is exactly $\log (1+y) / y \leqslant \frac{C}{y^{1-\frac{1}{\alpha}}}, \forall y \geqslant 0$, by compared growth.

Hence, we can deduce that for any $y \geqslant 0$ we have

$$
V(y) \leqslant \frac{C}{y^{1-\frac{1}{\alpha}}} .
$$

Combining (2.16) and (2.17), we deduce that for any $y \geqslant 0$,

$$
U(y)+V(y) \leqslant \frac{C}{y^{1-\frac{1}{\alpha}}} .
$$

Using (2.18) with $y=C|x| h^{\alpha} / m^{\alpha}$ together with (2.15), we obtain that

$$
\ln \left(Q_{m}^{1}(x)\right) \leqslant C h|x|^{\frac{1}{\alpha}} .
$$


Coming back to (2.14), we deduce that

$$
Q_{m}^{1}(x) \leqslant \exp \left(C h|x|^{\frac{1}{\alpha}}\right) .
$$

Now, if we restrict to the range $|x| \leqslant \frac{A}{h^{2 \alpha}}$, we deduce that $h|x|^{\frac{1}{\alpha}} \leqslant D_{A}|x|^{\frac{1}{2 \alpha}}$, so we have that

$$
Q_{m}^{1}(x) \leqslant D_{A} \exp \left(D_{A}|x|^{\frac{1}{2 \alpha}}\right) .
$$

- Second regime: $n+m \leqslant 1 / h$.

We use the following basic inequality, true for any $x \in(0, \pi / 2)$, any $y \in(0, \pi / 2-x)$, and any $\alpha \geqslant 1$ :

$$
\left|\sin (x)^{2 \alpha}-\sin (y)^{2 \alpha}\right| \geq\left(\left(\frac{2}{\pi}\right)^{2 \alpha}\left|x^{2 \alpha}-y^{2 \alpha}\right|\right) .
$$

We deduce that

$$
\left|\lambda_{n}^{2}-\lambda_{m}^{2}\right| \geqslant 2^{\alpha}\left|n^{2 \alpha}-m^{2 \alpha}\right| .
$$

Plugging this inequality into the second term from (2.12), we obtain that

$$
Q_{m}^{2}(x) \leqslant \prod_{\substack{1 \leq n \leq N \\ n \neq m}}\left(1+\frac{|x|}{\left|n^{2 \alpha}-m^{2 \alpha}\right|}\right) .
$$

Then,

$$
\begin{aligned}
\ln \left(Q_{m}^{2}(x)\right) & \leqslant \sum_{\substack{1 \leq n \leq N \\
n \neq m}} \ln \left(1+\frac{|x|}{\left|n^{2 \alpha}-m^{2 \alpha}\right|}\right) \\
& \leqslant \sum_{\substack{n \geq 1 \\
n \neq m}} \int_{0}^{|x|} \frac{d t}{t+\left|n^{2 \alpha}-m^{2 \alpha}\right|} \\
& \leqslant \int_{0}^{|x|} \sum_{\substack{1 \leq n \leq N \\
n \neq m}} \frac{d t}{t+\left|n^{2 \alpha}-m^{2 \alpha}\right|} \\
& \leqslant \int_{0}^{|x|} \sum_{\substack{1 \leq n \leq N \\
n \neq m}} \int_{\left|n^{2}-m^{2}\right|^{\alpha}}^{\infty} \frac{d s}{(t+s)^{2}} d t \\
& \leqslant \int_{0}^{|x|} \int_{\inf _{n}\left|n^{2 \alpha}-m^{2 \alpha}\right|}^{\sup _{n}\left|n^{2 \alpha}-m^{2 \alpha}\right|} \frac{L_{m}(s) d s}{(t+s)^{2}} d t \\
& \leqslant \int_{0}^{|x|} \int_{m^{2 \alpha-1}}^{\frac{1}{h^{2 \alpha}}} \frac{L_{m}(s) d s}{(t+s)^{2}} d t
\end{aligned}
$$

where

$$
L_{m}(s):=\operatorname{card}\left\{n \in \mathbb{N}^{*} \text { s.t. }\left|n^{2 \alpha}-m^{2 \alpha}\right| \leqslant s\right\} .
$$

Following the computations made in [27, Proof of Lemma 2.1], we can already infer that

$$
\ln \left(Q_{m}^{2}(x)\right) \leqslant C|x|^{\frac{1}{2 \alpha}},
$$

which gives (2.9) when combined with (2.11) and (2.20). 
Now, let us give another estimate on $Q_{m}^{2}$. It is easy to compute this counting function $L_{m}$. We have

$$
\left|n^{2 \alpha}-m^{2 \alpha}\right| \leqslant s \Leftrightarrow\left(\left(m^{2 \alpha}-s\right)^{+}\right)^{\frac{1}{2 \alpha}} \leqslant n \leqslant\left(m^{2 \alpha}+s\right)^{\frac{1}{2 \alpha}} .
$$

Hence, we deduce that

$$
\begin{aligned}
\ln \left(Q_{m}^{2}(x)\right) & \leqslant \int_{0}^{|x|} \int_{m^{2 \alpha-1}}^{\frac{1}{h^{2 \alpha}}} \frac{\left(m^{2 \alpha}+s\right)^{\frac{1}{2 \alpha}}-\left(\left(m^{2 \alpha}-s\right)^{+}\right)^{\frac{1}{2 \alpha}}}{(t+s)^{2}} d s d t \\
& \leqslant|x| \int_{m^{2 \alpha-1}}^{\frac{1}{h^{2 \alpha}}} \frac{\left(m^{2 \alpha}+s\right)^{\frac{1}{2 \alpha}}-\left(\left(m^{2 \alpha}-s\right)^{+}\right)^{\frac{1}{2 \alpha}}}{s(s+|x|)} d s .
\end{aligned}
$$

We introduce the change of variables $s=m^{2 \alpha} t$. We deduce that

$$
\ln \left(Q_{m}^{2}(x)\right) \leqslant \frac{|x|}{m^{2 \alpha-1}} \int_{\frac{1}{m}}^{\frac{1}{(m h)^{2 \alpha}}} \frac{(1+t)^{\frac{1}{2 \alpha}}-\left((1-t)^{+}\right)^{\frac{1}{2 \alpha}}}{t\left(t+\frac{|x|}{m^{2 \alpha}}\right)} d t .
$$

Let us introduce, for $y>0$,

$$
U(y)=\int_{\frac{1}{m}}^{1} \frac{(1+t)^{\frac{1}{2 \alpha}}-(1-t)^{\frac{1}{2 \alpha}}}{t(t+y)} d t \text { and } V(y)=\int_{1}^{\frac{1}{(h m)^{2 \alpha}}} \frac{(1+t)^{\frac{1}{2 \alpha}}}{t(t+y)} d t .
$$

Using the inequality $(1+t)^{\frac{1}{2 \alpha}}-(1-t)^{\frac{1}{2 \alpha}} \leqslant \alpha t$, true for any $x \in[0,1]$, we obtain

$$
U(y) \leqslant \int_{\frac{1}{m}}^{1} \frac{\alpha}{t+y} d t \leqslant \ln (m) .
$$

On the other hand, concerning $V$, we remark that

$$
V(y) \leqslant \int_{1}^{\infty} \frac{(1+t)^{\frac{1}{2 \alpha}}}{t^{2}} d t \leqslant C .
$$

Hence, using (2.22) together with (2.23) and (2.24), we deduce that

$$
\ln \left(Q_{m}^{2}(x)\right) \leqslant C \frac{|x| \ln (m)}{m^{2 \alpha-1}} .
$$

Finally, using (2.11) together with (2.19) and (2.25), we deduce estimate (2.10).

We see that contrary to the computations made for the wave equation in [26], the product $P_{m}$ is only bounded in the small range $|x| \leqslant C$. As soon as $x \geqslant \frac{C}{h^{s}}$ for some $s>0$, we have an upper bound that may explode as $h \rightarrow 0$.

Now, we consider 


$$
\begin{aligned}
R_{m}(z) & =\prod_{\substack{1 \leq|n| \leq N \\
n \neq m}}\left(\frac{z}{\lambda_{n}}-1\right) \prod_{\substack{1 \leq n \mid \leq N \\
n \neq m}} \frac{\lambda_{n}}{\lambda_{m}-\lambda_{n}} \\
& :=R_{m}^{1}(z) S_{m} \quad(z \in \mathbb{C}),
\end{aligned}
$$

where

$$
R_{m}^{1}(z)=\prod_{\substack{1 \leq|n| \leq N \\ n \neq m}}\left(\frac{z}{\lambda_{n}}-1\right)
$$

and

$$
S_{m}=\prod_{\substack{1 \leq|n| \leq N \\ n \neq m}} \frac{\lambda_{n}}{\lambda_{m}-\lambda_{n}}
$$

We remark that

$$
R_{m}\left(\lambda_{n}\right)=\delta_{n m}
$$

Moreover, $R_{m}$ is related to the product $P_{m}$ defined in (2.8) by the following relation:

$$
\left(1-\frac{x-\lambda_{m}}{\lambda_{-m}-\lambda_{m}}\right) P_{m}\left(x^{2}-\lambda_{m}^{2}\right)=R_{m}(x),
$$

so that our estimates on $P_{m}$ will give estimates on $R_{m}$. We start our estimates with the following technical result concerning the product $S_{m}$.

Lemma 2.2. For every $1 \leq|m| \leq N$, we have that

$$
\left|S_{m}\right| \leqslant \cos ^{2 \alpha} \frac{m \pi h}{2} .
$$

Proof of Lemma 2.2. From the symmetry of the sequence $\left(\lambda_{n}\right)_{1 \leq|n| \leq N}\left(\right.$ i.e. $\left.\lambda_{-n}=-\lambda_{n}\right)$, it is sufficient to consider only the case $1 \leq m \leq N$.

Firstly, we obtain that

$$
\left|S_{m}\right|=\prod_{\substack{1 \leq|n| \leq N \\ n \neq m}}\left|\frac{\lambda_{n}}{\lambda_{n}-\lambda_{m}}\right|=\frac{1}{2} \prod_{\substack{1 \leq n \leq N \\ n \neq m}}\left|\frac{\lambda_{n}^{2}}{\lambda_{n}^{2}-\lambda_{m}^{2}}\right|=\frac{1}{2} \prod_{\substack{1 \leq n \leq N \\ n \neq m}}\left|\frac{\sin ^{2 \alpha}\left(\frac{n \pi h}{2}\right)}{\sin ^{2 \alpha}\left(\frac{n \pi h}{2}\right)-\sin ^{2 \alpha}\left(\frac{m \pi h}{2}\right)}\right| .
$$

Since $\left|x^{\alpha}-y^{\alpha}\right| \geq|x-y|^{\alpha}$, for all $x, y \in \mathbb{R}$ and $\alpha>1$, it follows that

$$
\left|S_{m}\right| \leqslant \frac{1}{2} \prod_{\substack{1 \leq n \leq N \\ n \neq m}}\left|\frac{\sin ^{2}\left(\frac{n \pi h}{2}\right)}{\sin ^{2}\left(\frac{n \pi h}{2}\right)-\sin ^{2}\left(\frac{m \pi h}{2}\right)}\right|^{\alpha}=\frac{1}{2} \prod_{\substack{1 \leq n \leq N \\ n \neq m}}\left|\frac{\sin ^{2}\left(\frac{n \pi h}{2}\right)}{\sin \left(\frac{(m-n) \pi h}{2}\right) \sin \left(\frac{(m+n) \pi h}{2}\right)}\right|^{\alpha}
$$


Now we proceed similar to the wave equation (see [26]). We study the above product by splitting it into two parts. We have that

$$
\begin{gathered}
\prod_{\substack{1 \leq n \leq N \\
n \neq m}} \frac{\sin \left(\frac{n \pi h}{2}\right)}{\left|\sin \left(\frac{(n-m) \pi h}{2}\right)\right|}=\frac{\prod_{1 \leq n \leq m-1} \sin \left(\frac{n \pi h}{2}\right)}{\prod_{1 \leq n \leq m-1} \sin \left(\frac{n \pi h}{2}\right) \prod_{m+1 \leq n \leq N} \sin \left(\frac{n \pi h}{2}\right)} \\
=\frac{\prod_{1 \leq n \leq N-m} \sin \left(\frac{n \pi h}{2}\right)}{\prod_{k=m+1}^{N-m} \sin \left(\frac{k \pi h}{2}\right)}=\frac{\prod_{k=m+1}^{N} \sin \left(\frac{k \pi h}{2}\right)}{\prod_{k=1}^{N} \cos \left(\frac{k \pi h}{2}\right)}
\end{gathered}
$$

and also

$$
\begin{gathered}
\prod_{\substack{1 \leq n \leq N \\
n \neq m}} \frac{\sin \left(\frac{n \pi h}{2}\right)}{\sin \left(\frac{(n+m) \pi h}{2}\right)}=\frac{\sin \left(\frac{\pi h}{2}\right) \ldots \sin \left(\frac{N \pi h}{2}\right)}{\sin \left(\frac{m \pi h}{2}\right)} \frac{\sin \left(\frac{2 m \pi h}{2}\right)}{\sin \left(\frac{(m+1) \pi h}{2}\right) \ldots \sin \left(\frac{(m+N) \pi h}{2}\right)} \\
=2 \cos \left(\frac{m \pi h}{2}\right) \frac{\prod_{k=1}^{m} \sin \left(\frac{k \pi h}{2}\right)}{\prod_{k=N+1}^{m+N} \sin \left(\frac{k \pi h}{2}\right)}=2 \cos ^{2}\left(\frac{m \pi h}{2}\right) \frac{\prod_{k=1}^{m} \sin \left(\frac{k \pi h}{2}\right)}{\prod_{k=1}^{m} \cos \left(\frac{k \pi h}{2}\right)}
\end{gathered}
$$

where for the last estimate we have used the fact that $\sin \left(\frac{k \pi h}{2}\right)=\cos \left(\frac{(N+1-k) \pi h}{2}\right)$.

From the last two relations together with (2.29) we obtain that

$$
\left|S_{m}\right| \leqslant \cos ^{2 \alpha}\left(\frac{m \pi h}{2}\right)\left|\frac{\prod_{k=1}^{N} \sin \left(\frac{k \pi h}{2}\right)}{\prod_{k=1}^{N} \cos \left(\frac{k \pi h}{2}\right)}\right|^{\alpha}=\cos ^{2 \alpha}\left(\frac{m \pi h}{2}\right),
$$

and the proof is complete.

Now, let us study the product $R_{m}$ for high values of $|x|$.

First of all, let us rewrite $R_{m}^{1}$ as

$$
R_{m}^{1}(z)=\frac{z+\lambda_{m}}{\lambda_{m}} \prod_{\substack{1 \leq n \leq N \\ n \neq m}}\left(\frac{z^{2}}{\lambda_{n}^{2}}-1\right) .
$$

Proposition 2.3. There exist a constant $R \in \mathbb{N}^{*}$ such that for $N$ large enough and for $|x| \geqslant\left(\frac{2}{h}\right)^{\alpha}$, we have

$$
\left|R_{m}(x)\right| \leqslant\left(|x| h^{\alpha}\right)^{\frac{R}{h}} .
$$


Proof of Proposition 2.3 Since $|x| \geqslant\left(\frac{2}{h}\right)^{\alpha}$, let us denote by $r=\left(\frac{x h^{\alpha}}{2^{\alpha}}\right)^{2} \geqslant 1$. Then,

$$
\begin{gathered}
\left|R_{m}^{1}(x)\right|=\prod_{\substack{1 \leq n \leq N \\
n \neq m}}\left|\frac{x^{2}}{\lambda_{n}^{2}}-1\right|=\prod_{\substack{1 \leq n \leq N \\
n \neq m}}\left|\frac{r}{\sin ^{2 \alpha}\left(\frac{n \pi h}{2}\right)}-1\right|=\prod_{\substack{1 \leq n \leq N \\
n \neq N+1-m}}\left|\frac{r}{\cos ^{2 \alpha}\left(\frac{n \pi h}{2}\right)}-1\right| \\
=\frac{\cos ^{2 \alpha}\left(\frac{(N+1-m) \pi h}{2}\right)}{r-\cos ^{2 \alpha}\left(\frac{(N+1-m) \pi h}{2}\right)} \prod_{1 \leq n \leq N}\left(\frac{r}{\cos ^{2 \alpha}\left(\frac{n \pi h}{2}\right)}-1\right) \\
\leqslant \frac{\cos ^{2 \alpha}\left(\frac{(N+1-m) \pi h}{2}\right)}{r-\cos ^{2 \alpha}\left(\frac{(N+1-m) \pi h}{2}\right)} \exp \left(\int_{1}^{N} \ln \left(\frac{r}{\cos ^{2 \alpha}\left(\frac{t \pi h}{2}\right)}-1\right) d t\right) .
\end{gathered}
$$

In what follows we compute the last integral by using a change of variable $u=\tan \frac{t \pi h}{2}$ :

$$
\int_{1}^{N} \ln \left(\frac{r}{\cos ^{2 \alpha}\left(\frac{t \pi h}{2}\right)}-1\right) d t=\frac{2}{\pi h} \int_{C h}^{\infty} \frac{\ln \left(r\left(1+u^{2}\right)^{\alpha}-1\right)}{1+u^{2}} d u .
$$

Note that the following inequality holds

$$
\ln \left(r\left(1+u^{2}\right)^{\alpha}-1\right) \leqslant C \ln (r+1)\left(1+u^{2}\right)^{\frac{1}{4 \alpha}} \quad(r \geqslant 1, u \geqslant 0),
$$

so that we obtain

$$
\begin{aligned}
& \int_{C h}^{\infty} \frac{\ln \left(r\left(1+u^{2}\right)^{\alpha}-1\right)}{1+u^{2}} d u \leqslant C \ln (r+1) \int_{C h}^{\infty} \frac{1}{\left(1+u^{2}\right)^{1-1 /(4 \alpha)}} d u \\
& \leqslant C \ln (r+1) .
\end{aligned}
$$

Using (2.26) together with (2.28) and the previous estimate, we deduce that for ant $|x| \geqslant\left(\frac{2}{h}\right)^{\alpha}$, we have

$$
\left|R_{m}(x)\right| \leqslant \exp \left(\frac{C}{h} \ln \left(\frac{x h^{\alpha}}{2^{\alpha}}+1\right)\right) \leqslant \exp \left(\frac{C}{h} \ln \left(\frac{2 x h^{\alpha}}{2^{\alpha}}\right)\right) .
$$

Hence the proof of the estimate of $R_{m}$ given in (2.30) is finished, taking $R=\lceil C\rceil$.

Remark 2.2. Let $A>0$. By using Proposition 2.2, (2.27) and Proposition 2.3, we infer the existence of two constants $R \in \mathbb{N}^{*}$ and $C>0$ such that for $N$ large enough we have

$$
\left|R_{m}(x)\right| \leqslant \varphi(x)
$$

where

$$
\varphi(x)= \begin{cases}D_{A} \exp \left(D_{A}\left|x^{2}-\lambda_{m}^{2}\right|^{\frac{1}{2 \alpha}}\right) & |x| \leq \frac{A}{h^{2 \alpha}} \\ \left(|x| h^{\alpha}\right)^{\frac{R}{h}} & |x| \geq\left(\frac{2}{h}\right)^{\alpha} \\ C \exp \left(C\left(h\left|x^{2}-\lambda_{m}^{2}\right|^{\frac{1}{\alpha}}+\frac{\left|x^{2}-\lambda_{m}^{2}\right| \ln (m)}{m^{2 \alpha-1}}\right)\right) & x \in \mathbb{R}\end{cases}
$$




\subsection{Construction of the multiplier}

To get our multiplier, taking into account the different bad behaviour of the product $R_{M}$ that requires to be compensated on the real line (see Remark 2.2), we need to consider a construction in two parts. Let us remind that $2 / h=2(N+1) \in \mathbb{N}$. Hence, we use a new construction, mixing a part similar to the construction of [26] and another part, which is similar to the construction made in [29].

For every $b>0$ we set

$$
H_{b}:=\frac{1_{[-b, b]}}{2 b} .
$$

We remark that

$$
\int_{\mathbb{R}} H_{b}=\int_{-b}^{b} H_{b}=1
$$

We denote by

$$
a:=\frac{h}{2 R},
$$

so that

$$
\sum_{n=1}^{R / h} a=\frac{1}{2} .
$$

Remind that $R$ was introduced in Proposition 2.3. We consider the product

$$
u_{a}:=H_{a} * \ldots * H_{a},
$$

where $*$ represents the convolution product. The product is made of $R / h$ terms.

We introduce

$$
M_{m}^{1}(z):=\int_{-1 / 2}^{1 / 2} u_{a}(t) e^{-i \frac{T}{2}\left(z-\lambda_{m}\right) t} d t .
$$

One has the following properties on $M_{m}^{1}$ (see [26, Lemma 2.6]):

Lemma 2.3. $M_{m}^{1}$ is of exponential type T/4. Moreover, one has

$$
M_{m}^{1}\left(\lambda_{m}\right)=1
$$

and for every $x \in \mathbb{R}$,

$$
\left|M_{m}^{1}(x)\right| \leqslant 1
$$

Moreover,

$$
\left|M_{m}^{1}(x)\right| \leqslant\left(\frac{4 R}{\left|x-\lambda_{m}\right| T h}\right)^{\frac{R}{h}} .
$$

Now, we consider the second part of the multiplier. Let $\nu>0$ be some large parameter to be chosen later. Let also $\gamma \in(1, \alpha)$ some parameter to be chosen later close enough to 1 . We consider

$$
\sigma_{\nu}:=\lim _{n \rightarrow \infty} H_{a_{0}} * H_{a_{1}} * \cdots * H_{a_{n}},
$$


where the sequence $\left(a_{k}\right)_{k \in \mathbb{N}}$ is given by

$$
\begin{cases}a_{k}=\frac{1}{\nu} & \text { if } k \leqslant\lfloor\nu\rfloor, \\ a_{k}=\frac{\nu^{\gamma-1}}{k^{\gamma}} & \text { if } k \geqslant\lfloor\nu\rfloor+1 .\end{cases}
$$

We consider the corresponding function $\sigma_{\nu}$ constructed as before from the sequence $\left(a_{k}\right)_{k \in \mathbb{N}}$. We call $a(\nu)$ its support, that is to say

$$
a(\nu):=\sum_{k=0}^{\infty} a_{k}=\frac{\lfloor\nu\rfloor+1}{\nu}+\sum_{k=\lfloor\nu\rfloor+1}^{\infty} \frac{\nu^{\gamma-1}}{k^{\gamma}} .
$$

Taking into account the definition of $a(\nu)$, we may observe that there exists some constant $S(\gamma)>0$ (not depending on $\nu$ ) such that

$$
a(\nu) \leqslant S(\gamma)
$$

Now, we introduce

$$
H_{\beta}(z):=\int_{-a(\nu)}^{a(\nu)} \sigma_{\nu}(t) e^{-i \beta t z} d t
$$

We have the following properties ([29, Lemma 3.4]).

Lemma 2.4. The following estimates hold:

$$
\begin{aligned}
H_{\beta}(0) & =1 \\
\left|H_{\beta}(z)\right| & \leqslant e^{S(\gamma) \beta|\operatorname{Im}(z)|}, \\
\left|H_{\beta}(x)\right| & \leqslant C \nu e^{\gamma \nu} e^{-\frac{\gamma}{2}\left(\left(\beta \nu^{\gamma-1}\right)|x|\right)^{1 / \gamma}}, x \in \mathbb{R} .
\end{aligned}
$$

Firstly, we choose $\beta=\frac{S(\gamma) T}{4}$, so that by (2.35) and (2.37), $H_{\beta}$ is of exponential type $T / 4$. We also consider any $K>0$ (to be chosen later) and we choose $\nu$ such that

$$
\frac{\alpha}{2}\left(\beta \nu^{\gamma-1}\right)^{\frac{1}{\gamma}}=K \text {. }
$$

Remark that $\nu$ is only depending on $\beta, T$ and $K$, but not on $h$. Hence, from (2.38) we can write the final useful estimate

$$
\left|H_{\beta}(x)\right| \leqslant C e^{-K|x|^{\frac{1}{\gamma}}}, x \in \mathbb{R},
$$

where $C$ depend on $T, \gamma$ and $K$ but not on $h$. Our second part of the multiplier will then be $M_{m}^{2}(z)=H_{\beta}\left(x-\lambda_{n}\right)$.

\subsection{Construction and estimation of the biorthogonal family}

We can now introduce the family

$$
\psi_{m}(z)=R_{m}(z) M_{m}^{1}(z) M_{m}^{2}(z) .
$$

Let us study this product in different regimes, using the previous estimates. We assume without loss of generality that $\lambda_{m} \geqslant 0$. 
1. If $|x| \leqslant\left(\frac{2}{h}\right)^{\alpha}$, then there exists some $A>0$ such that for any such $x$ and any $\lambda_{m}$, we have

$$
\left|x^{2}-\lambda_{m}^{2}\right| \leqslant\left(\frac{A}{h}\right)^{2 \alpha}
$$

(one may for example take $A=4$ ).

Let us consider different subranges of $x$.

- Assume that $\left|x-\lambda_{m}\right| \geqslant \lambda_{m}^{\delta}$ for some $\delta \in(0,1)$ to be chosen. We can use now (2.27), (2.9), (2.33) and (2.39) to obtain

$$
\left|\psi_{m}(x)\right| \leqslant C(1+|x|) D_{A} e^{D_{A}\left|x^{2}-\lambda_{m}^{2}\right|^{\frac{1}{2 \alpha}}-K\left|x-\lambda_{m}\right|^{\frac{1}{\gamma}}} .
$$

We want to choose $\delta$ such that there exists some $K^{\prime}>0$ (depending on $\delta, K, \alpha$ and $\gamma$ ) such that for any $x$ such that $\left|x-\lambda_{m}\right| \geqslant \lambda_{m}^{\delta}$, we have

$$
D_{A}\left|x^{2}-\lambda_{m}^{2}\right|^{\frac{1}{2 \alpha}}-K\left|x-\lambda_{m}\right|^{\frac{1}{\gamma}} \leqslant-K^{\prime}\left|x^{2}-\lambda_{m}^{2}\right|^{\frac{1}{2 \alpha}} .
$$

this is equivalent to

$$
\left(D_{A}+K^{\prime}\right)\left|x^{2}-\lambda_{m}^{2}\right|^{\frac{1}{2 \alpha}} \leqslant K\left|x-\lambda_{m}\right|^{\frac{1}{\gamma}-\frac{1}{2 \alpha}}
$$

i.e.

$$
\left(D_{A}+K^{\prime}\right)\left|x+\lambda_{m}\right|^{\frac{1}{2 \alpha}} \leqslant K\left|x-\lambda_{m}\right|^{\frac{1}{\gamma}-\frac{1}{2 \alpha}} .
$$

Assume in addition for the moment that $\left|x-\lambda_{m}\right| \geqslant \lambda_{m}$. Then, one readily verifies that there exists some constant $C>0$ (independent on $m, x$ or $h$ ) such that for any $x$ such that $\left|x-\lambda_{m}\right| \geqslant \lambda_{m}$, we have

$$
\frac{\left|x+\lambda_{m}\right|^{\frac{1}{2 \alpha}}}{\left|x-\lambda_{m}\right|^{\frac{1}{\gamma}-\frac{1}{2 \alpha}}} \leqslant C,
$$

for some constant $C>0$. Hence, we deduce that (2.42) is automatically verified for some $K^{\prime}>0$ as soon as $\gamma<\alpha$ (which is true) and $K$ is sufficiently large, which is assumed from now on. Assume now that $\left|x-\lambda_{m}\right| \leqslant \lambda_{m}$, which implies notably that $\left|x+\lambda_{m}\right| \leqslant 3 \lambda_{m}$. In this case, taking into account that $\left|x-\lambda_{m}\right| \geqslant \lambda_{m}^{\delta}$, we see that (2.42) is verified for some $K^{\prime}>0$ as soon as $\delta\left(\frac{1}{\gamma}-\frac{1}{2 \alpha}\right)>\frac{1}{2 \alpha}$ and $K$ is chosen large enough. Taking into account that we also want $\delta<1$, we deduce that we require

$$
1>\delta>\frac{\gamma}{2 \alpha-\gamma}
$$

which is possible since $\gamma<\alpha$.

To summarize, under condition (2.43), we have the existence of some $K^{\prime}>0$ (depending on $\gamma, \delta$ and $\alpha$ ) such that (2.41) holds as soon as $\left|x-\lambda_{m}\right| \geqslant \lambda_{m}^{\delta}$ and $K$ is chosen large enough, which is assumed from now on.

- Assume now that $\left|x-\lambda_{m}\right| \leqslant \lambda_{m}^{\delta}$ for $\delta$ verifying (2.43). In this case, we use (2.27), (2.10), (2.33) and (2.39) to obtain

$$
\left|\psi_{m}(x)\right| \leqslant C(1+|x|) e^{C\left(h\left|x^{2}-\lambda_{m}^{2}\right|^{\frac{1}{\alpha}}+\frac{\left|x^{2}-\lambda_{m}^{2}\right| \ln (m)}{m^{2 \alpha-1}}\right)-K\left|x-\lambda_{m}\right|^{\frac{1}{\gamma}}} .
$$

Hence, we notably have 


$$
\left|\psi_{m}(x)\right| \leqslant C e^{C\left(h\left|x^{2}-\lambda_{m}^{2}\right| \frac{1}{\alpha}+\frac{\left|x^{2}-\lambda_{m}^{2}\right| \ln \left(\lambda_{m}\right)}{\lambda_{m}^{2-\frac{1}{\alpha}}}\right)-K\left|x-\lambda_{m}\right|^{\frac{1}{\gamma}}} .
$$

We remark that since we chose $\delta<1$, we have $\left|x+\lambda_{m}\right| \leqslant C \lambda_{m}$, so that

$$
h\left|x^{2}-\lambda_{m}^{2}\right|^{\frac{1}{\alpha}} \leqslant C h \lambda_{m}^{\frac{1}{\alpha}}\left|x-\lambda_{m}\right|^{\frac{1}{\alpha}} .
$$

Hence, taking into account that $\lambda_{m} \leqslant \frac{C}{h^{\alpha}}$, we have

$$
h\left|x^{2}-\lambda_{m}^{2}\right|^{\frac{1}{\alpha}} \leqslant C\left|x-\lambda_{m}\right|^{\frac{1}{\alpha}} .
$$

We deduce the existence of some $K^{\prime}>0$ such that

$$
h\left|x^{2}-\lambda_{m}^{2}\right|^{\frac{1}{\alpha}}-K\left|x-\lambda_{m}\right|^{\frac{1}{\gamma}} \leqslant-K^{\prime}\left|x-\lambda_{m}\right|^{\frac{1}{\alpha}}
$$

as soon as $K$ is chosen large enough, which is assumed from now on.

Concerning the other term, since we chose $\delta<1$, we have $\left|x+\lambda_{m}\right| \leqslant C \lambda_{m}$, hence

$$
\begin{aligned}
\frac{\left|x^{2}-\lambda_{m}^{2}\right| \ln \left(\lambda_{m}\right)}{\lambda_{m}^{2-\frac{1}{\alpha}}}-K\left|x-\lambda_{m}\right|^{\frac{1}{\gamma}} & \leqslant C \frac{\left|x-\lambda_{m}\right| \ln \left(\lambda_{m}\right)}{\lambda_{m}^{1-\frac{1}{\alpha}}}-K\left|x-\lambda_{m}\right|^{\frac{1}{\gamma}} \\
& \leqslant\left|x-\lambda_{m}\right|^{\frac{1}{\gamma}}\left(C \frac{\left|x-\lambda_{m}\right|^{1-\frac{1}{\gamma}} \ln \left(\lambda_{m}\right)}{\lambda_{m}^{1-\frac{1}{\alpha}}}-K\right) \\
& \leqslant\left|x-\lambda_{m}\right|^{\frac{1}{\gamma}}\left(C \frac{\lambda_{m}^{\delta\left(1-\frac{1}{\gamma}\right)} \ln \left(\lambda_{m}\right)}{\lambda_{m}^{1-\frac{1}{\alpha}}}-K\right) .
\end{aligned}
$$

We deduce the existence of some $K^{\prime \prime}>0$ such that

$$
\frac{\left|x^{2}-\lambda_{m}^{2}\right| \ln \left(\lambda_{m}\right)}{\lambda_{m}^{2-\frac{1}{\alpha}}}-K\left|x-\lambda_{m}\right|^{\frac{1}{\gamma}} \leqslant-K^{\prime}\left|x-\lambda_{m}\right|^{\frac{1}{\gamma}}
$$

as soon as $K$ is chosen large enough (which is assumed from now on) and $\delta$ is chosen such that

$$
\delta\left(1-\frac{1}{\gamma}\right)<\left(1-\frac{1}{\alpha}\right)
$$

i.e.

$$
\delta<\frac{\gamma(\alpha-1)}{\alpha(\gamma-1)}
$$

Satisfying both (2.43) and (2.46) is only possible if we have

$$
\frac{\gamma}{2 \alpha-\gamma}<\frac{\gamma(\alpha-1)}{\alpha(\gamma-1)}
$$

which is equivalent to

$$
\alpha(\gamma-1)<(2 \alpha-\gamma)(\alpha-1) .
$$

This last inequality is true since $\gamma \in(1, \alpha)$. 
2. If $|x| \geqslant\left(\frac{2}{h}\right)^{\alpha}$, then we use (2.30), (2.34) and (2.39), with $K>0$ and $\gamma>0$ as in the previous point. Taking into account that $\gamma<\alpha$ and $\left|x^{2}-\lambda_{m}^{2}\right| \geqslant C$ for some $C>0$ (independent of $m$ but depending on $\Gamma$ ), we obtain

$$
\begin{gathered}
\left|\psi_{m}(x)\right| \leqslant C e^{-2 K\left|x^{2}-\lambda_{m}^{2}\right|^{\frac{1}{2 \gamma}}}\left(|x| h^{\alpha}\right)^{\frac{R}{h}}\left(\frac{4 R}{\left|x-\lambda_{m}\right| T h}\right)^{\frac{R}{h}} \\
\leqslant C D_{A} e^{-2 D_{A}\left|x^{2}-\lambda_{m}^{2}\right|^{\frac{1}{2 \alpha}}}\left(\frac{4 R|x| h^{\alpha}}{\left|x-\lambda_{m}\right| T h}\right)^{\frac{R}{h}} .
\end{gathered}
$$

Using that $|m| \leqslant f(N)$, we easily deduce as in [26, Proof of Prop. 2.3] that there exists some $C_{1}>0$ such that

$$
\frac{|x|}{\left|x-\lambda_{m}\right|} \leqslant C_{1}
$$

Hence, using now that $\alpha>1$, we deduce that for some $K^{\prime}>0$,

$$
\left|\psi_{m}(x)\right| \leqslant C e^{-C\left|x^{2}-\lambda_{m}^{2}\right|^{\frac{1}{2 \alpha}}}\left(\frac{4 R C_{1} h^{\alpha-1}}{T}\right)^{\frac{R}{h}} \leqslant C e^{-K^{\prime}\left|x^{2}-\lambda_{m}^{2}\right|^{\frac{1}{2 \alpha}}} .
$$

In any cases, combining (2.41), (2.44), (2.45) and (2.47), we have, for any $x \in \mathbb{R}$, the following estimate:

$$
\left|\psi_{m}(x)\right| \leqslant C \max \left\{e^{-C\left|x^{2}-\lambda_{m}^{2}\right|^{\frac{1}{2 \alpha}}}, e^{-C\left|x-\lambda_{m}\right|^{\frac{1}{\gamma}}}, e^{-C\left|x-\lambda_{m}\right|^{\frac{1}{\alpha}}}\right\} .
$$

Hence, $\psi_{m} \in L^{2}(\mathbb{R})$ and is of exponential type at most $T / 2$ thanks to $(2.37),(2.31)$ and the fact that $P_{m}$ is obviously of exponential type 0 . We can use the Paley-Wiener Theorem (see e.g. [40, Theorem 19.3, Page 370] and we introduce the Fourier transform $\theta_{m} \in L^{2}(0, T)$ to $\psi_{m}$, which verifies, thanks to the definition of $R_{m}$ given in (2.26), (2.36) and (2.32), that $\theta_{m}\left(\lambda_{n}\right)=\delta_{n m}$ and is compactly supported in $[-T / 2, T / 2]$.

\section{Proofs of Theorem 1.1 and Corollary 1}

\subsection{Proof of Theorem 1.1}

\section{Proof of Theorem 1.1.}

Let $\left(\Theta_{m}\right)_{1 \leq|m| \leq N}$ be the biorthogonal sequence to the family $\left(e^{i \lambda_{n} t}\right)_{1 \leq|n| \leq N}$ in $L^{2}\left(-\frac{T}{2}, \frac{T}{2}\right)$ constructed and evaluated in Section 2.4 as the Fourier transform of $\psi_{m}$ introduced in (2.40), verifying by definition

$$
\int_{-\frac{T}{2}}^{\frac{T}{2}} \Theta_{m}(t) e^{i \lambda_{n} t} d t=\delta_{m n} \quad(|m|,|n|=1, \ldots, N) .
$$

According to Lemma 2.1 an more precisely identity (2.7), we consider the control function $v_{h} \in$ $\left(L^{2}(0, T)\right)^{N}$ given by

$$
v_{h}(t)=\sum_{1 \leq|n| \leq f(N)} \frac{a_{n} B_{h}^{n}}{\left\|B_{h}^{n}\right\|^{2}} e^{-i \lambda_{n} \frac{T}{2}} \Theta_{n}\left(t-\frac{T}{2}\right) .
$$


where $B_{h}^{n}$ is given by

$$
B_{h}^{n}=h\left(\chi_{(a, b)}(j h) \sin (j \pi n h)\right)_{1 \leq j \leq N} .
$$

Let us prove that here exists a constant $C>0$ (independent of $n$ or $N$ ) such that for $h$ small enough (depending on $a$ and $b$ ), we have

$$
\left\|B_{h}^{n}\right\|^{2} \geqslant C h
$$

First of all, we remark that

$$
\begin{aligned}
& \left.h \sum_{j=1}^{N}\left(\chi_{(a, b)}(j h) \sin (j \pi n h)\right)\right)^{2}=h \sum_{j=\left\lceil\frac{a}{h}\right\rceil}^{\left\lfloor\frac{b}{h}\right\rfloor} \sin (j \pi n h)^{2} \\
& =\frac{h}{4}\left(2+\left\lfloor\frac{2 b}{h}\right\rfloor-\left\lceil\frac{2 a}{h}\right\rceil+\frac{\sin \left(2 h\left\lceil\frac{a}{h}\right\rceil n \pi-h n \pi\right)-\sin \left(2 h\left\lfloor\frac{b}{h}\right\rfloor n \pi+h n \pi\right)}{\sin (n \pi h)}\right) .
\end{aligned}
$$

Let us consider two different cases:

- Assume that $n$ is such that $\liminf _{h \rightarrow 0} n h>0$. In this case, since $n \leqslant f(N)$, using Hypothesis (1.5), we have that there exists $C>0$ such that for any $h>0, \sin (n \pi h) \geqslant C$. We deduce that

$$
\begin{aligned}
& \frac{h}{4}\left|\frac{\sin \left(2 h\left\lceil\frac{a}{h}\right\rceil n \pi-h n \pi\right)-\sin \left(2 h\left\lfloor\frac{b}{h}\right\rfloor n \pi+h n \pi\right)}{\sin (n \pi h)}\right| \\
& \leqslant C \frac{h}{4}\left|\sin \left(2 h\left\lceil\frac{a}{h}\right\rceil n \pi-h n \pi\right)-\sin \left(2 h\left\lfloor\frac{b}{h}\right\rfloor n \pi+h n \pi\right)\right| \\
& \leqslant 2 C \frac{h}{4}
\end{aligned}
$$

which goes to 0 as $h \rightarrow 0$. Hence, we deduce that in this case

$$
h \sum_{j=1}^{N}\left(\chi_{(a, b)}(j h) \sin (j \pi n h)\right)^{2} \rightarrow \frac{b-a}{2} \text { as } h \rightarrow 0 .
$$

- Assume now that $n$ is such that $\liminf _{h \rightarrow 0} n h=0$. In this case, we have

$$
\left.\liminf _{h \rightarrow 0} h \sum_{j=1}^{N}\left(\chi_{(a, b)}(j h) \sin (j \pi n h)\right)\right)^{2}=\frac{b-a}{2}+\frac{\sin (2 a \pi)-\sin (2 b \pi)}{4 \pi} .
$$

Moreover, it is very easy to prove that

$$
\frac{b-a}{2}+\frac{\sin (2 a \pi)-\sin (2 b \pi)}{4 \pi}>0
$$

as soon as $a \neq b$. Indeed, if $\frac{b-a}{2}=\frac{\sin (2 b \pi)-\sin (2 a \pi)}{4 \pi}$, then we have $2 \pi b-\sin (2 b \pi)=2 \pi a-$

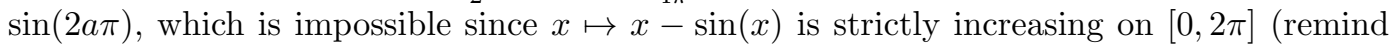
that $0<a<b<1)$.

Hence, we have the existence of $C>0$ such that whatever $h \in(0,1)$ and $n \in[|0, f(N)|]$ are, we have

$$
\left.h \sum_{j=1}^{N}\left(\chi_{(a, b)}(j h) \sin (j \pi n h)\right)\right)^{2} \geqslant C .
$$


This implies that (3.3) is verified.

Finally, we can prove the uniformly boundedness of the controls sequence $\left(v_{h}\right)_{h>0}$. Indeed, using the definition of $v_{h}$ given in (3.1) together with (3.3), (2.48) and the fact that the Fourier transform is an isometry in $L^{2}(\mathbb{R})$, we deduce that there exists $C_{1}>0$ such that

$$
\begin{aligned}
& \left\|v_{h}\right\|_{L^{2}}^{2} \leq C_{1} \sum_{1 \leq|n| \leq f(N)} \sum_{1 \leq|m| \leq f(N)} \frac{h^{2}\left|a_{n}\right|\left|a_{m}\right| \chi_{(a, b)}(j h) \sin (j \pi n h) \sin (j \pi m h) \mid}{h^{2}} \int_{\mathbb{R}}\left|\psi_{n}(x) \psi_{m}(x)\right| d x \\
& \leqslant C_{1} \sum_{1 \leq|n| \leq f(N)} \sum_{1 \leq|m| \leq f(N)}\left|a_{n}\right|\left|a_{m}\right| \max \left\{\int_{\mathbb{R}} e^{-C\left|x^{2}-\lambda_{n}^{2}\right|^{\frac{1}{2 \alpha}}-C\left|x^{2}-\lambda_{m}^{2}\right|^{\frac{1}{2 \alpha}}} d x,\right. \\
& \left.\int_{\mathbb{R}} e^{-C\left|x-\lambda_{n}\right|^{\frac{1}{\gamma}}-C\left|x-\lambda_{m}\right|^{\frac{1}{\gamma}}}, d x, \int_{\mathbb{R}} e^{-C\left|x-\lambda_{n}\right|^{\frac{1}{\alpha}}-C\left|x-\lambda_{m}\right|^{\frac{1}{\alpha}}}\right\},
\end{aligned}
$$

where $C$ is the constant from (2.48).

Consider some $|m| \geqslant|n|$ (otherwise, the same reasoning applies by exchanging $n$ and $m$ ). Then, we make the change of variables $x^{2}=y^{2}+\lambda_{m}^{2}$. Since $\lambda_{m}^{2}-\lambda_{n}^{2} \geqslant 0$, we obtain that

$$
\begin{aligned}
\int_{\mathbb{R}} e^{-C\left|x^{2}-\lambda_{n}^{2}\right|^{\frac{1}{2 \alpha}}-C\left|x^{2}-\lambda_{m}^{2}\right|^{\frac{1}{2 \alpha}}} & =\int_{\mathbb{R}} \frac{|y|}{\sqrt{y^{2}+\lambda_{m}^{2}}} e^{-C\left|y^{2}+\lambda_{m}^{2}-\lambda_{n}^{2}\right|^{\frac{1}{2 \alpha}}-C|y|^{\frac{1}{\alpha}}} d y \\
& \leqslant C \int_{\mathbb{R}} e^{-C\left|\lambda_{m}^{2}-\lambda_{n}^{2}\right|^{\frac{1}{2 \alpha}}-C|y|^{\frac{1}{\alpha}}} d y \leqslant C e^{-C\left|\lambda_{m}^{2}-\lambda_{n}^{2}\right|^{\frac{1}{2 \alpha}}} .
\end{aligned}
$$

It is very easy to prove that there exists some $C>0$ (that may depend on $\alpha$ ) such we have

$$
e^{-C\left|\lambda_{m}^{2}-\lambda_{n}^{2}\right|^{\frac{1}{2 \alpha}}} \leqslant \frac{C}{\left(1+\left|\lambda_{m}^{2}-\lambda_{n}^{2}\right|^{\frac{2}{\alpha}}\right)} .
$$

From (2.13) and (2.21), we deduce that there exists some $C>0$ such that

$$
\left|\lambda_{n}^{2}-\lambda_{m}^{2}\right|^{\frac{2}{\alpha}} \geqslant C|n-m|^{2}
$$

from which we obtain

$$
e^{-C\left|\lambda_{m}^{2}-\lambda_{n}^{2}\right|^{\frac{1}{2 \alpha}}} \leqslant \frac{C}{1+C|n-m|^{2}}
$$

By the same type of arguments, we can also easily obtain the estimates

$$
\int_{\mathbb{R}} e^{-C\left|x-\lambda_{n}\right|^{\frac{1}{\gamma}}-C\left|x-\lambda_{m}\right|^{\frac{1}{\gamma}}} \leqslant C e^{-C\left|\lambda_{m}-\lambda_{n}\right|^{\frac{1}{\gamma}}}, \int_{\mathbb{R}} e^{-C\left|x-\lambda_{n}\right|^{\frac{1}{\alpha}}-C\left|x-\lambda_{m}\right|^{\frac{1}{\alpha}}} \leqslant C e^{-C\left|\lambda_{m}-\lambda_{n}\right|^{\frac{1}{\alpha}}},
$$

and

$$
e^{-C\left|\lambda_{m}-\lambda_{n}\right|^{\frac{1}{\gamma}}} \leqslant \frac{C}{1+C|n-m|^{2}}, e^{-C\left|\lambda_{m}-\lambda_{n}\right|^{\frac{1}{\alpha}}} \leqslant \frac{C}{1+C|n-m|^{2}}
$$

Combining (3.4), (3.5), (3.6), (3.7), (3.8) and Young's inequality, we deduce that 


$$
\begin{aligned}
\int_{0}^{T}\left|v_{h}(t)\right|^{2} d t & \leqslant C \sum_{1 \leq|n| \leq f(N)} \sum_{1 \leq|m| \leq f(N)} \frac{\left|a_{n}\right|\left|a_{m}\right|}{1+C|n-m|^{2}} \\
& \leqslant C \sum_{1 \leq|n| \leq f(N)} \sum_{1 \leq|m| \leq f(N)} \frac{\left(\left|a_{n}\right|^{2}+\left|a_{m}\right|^{2}\right)}{\left(1+C|n-m|^{2}\right)^{2}} \\
& \leqslant C \sum_{1 \leq|n| \leq f(N)}\left|a_{n}\right|^{2}\left(\sum_{1 \leq|m| \leq f(N)} \frac{1}{\left(1+C|n-m|^{2}\right)^{2}}\right) \\
& \leqslant C \sum_{1 \leq|n| \leq f(N)}\left|a_{n}\right|^{2},
\end{aligned}
$$

and the proof of Theorem 1.1 is finished.

\subsection{Proof of Corollary 1}

The aim of this subsection is to prove the convergence of the controls sequence $\left(v_{h}\right)_{h>0}$ to a control of the continuous problem (1.1).

We study the existence of a control function $v \in L^{2}((0, T) \times(0,1))$ such that the solution of equation (1.1) verifies

$$
u(T)=u^{\prime}(T)=0
$$

We transform the controllability of the continuous problem into a moment problem, using an additional variational lemma.

Lemma 3.1. Problem (1.1) is controllable if and only if, for any initial datum $\left(u^{0}, u^{1}\right) \in \mathcal{H}$ decomposed as in (1.2), there exists $v \in L^{2}((0, T) \times(0,1))$ such that

$$
\left\langle\bar{w}^{1}, u^{0}\right\rangle_{D\left(\left(-\Delta_{D}\right)^{\alpha / 2}\right)^{\prime}, D\left(\left(-\Delta_{D}\right)^{\alpha / 2}\right)}-\int_{0}^{1} u^{1}(x) \bar{w}^{0}(x) d x=\int_{a}^{b} \int_{0}^{T} v(t, x) \bar{w}(t, x) d t d x,
$$

for all $\left(w^{0}, w^{1}\right) \in \mathcal{H}^{\prime}=L^{2}(0,1) \times D\left(\left(-\Delta_{D}\right)^{\alpha / 2}\right)^{\prime}$, where $w$ is the the solution of the adjoint problem

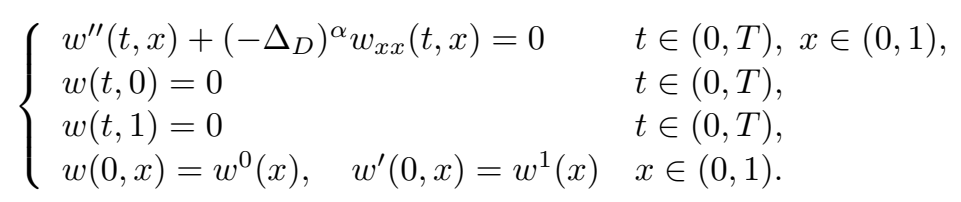

Proof of Lemma 3.1. Assume that we have $\left(u^{0}, u^{1}\right) \in D\left(\left(-\Delta_{D}\right)^{\alpha / 2+1 / 2}\right) \times H^{1}(0,1)$ and $\left(w^{0}, w^{1}\right) \in D\left(\left(-\Delta_{D}\right)^{\alpha / 2+1 / 2}\right) \times H^{1}(0,1)$. Multiplying (1.1) with $\bar{w}$ and integrating by parts from 0 to $T$ we have

$$
\left.\left(u^{\prime} \bar{w}-u \bar{w}^{\prime}\right)\right|_{0} ^{T}+\int_{0}^{T} u\left(\bar{w}^{\prime \prime}+\left(-\Delta_{D}\right)^{\alpha} \bar{w}\right) d t=\int_{0}^{T} v(t, x) \chi_{(a, b)}(x) \bar{w}(x, t) d t .
$$


Finally, if we integrate in space interval $[0,1]$ and taking into account the hypotheses and the fact that (1.1) is controllable if and only if $u(T)=u^{\prime}(T)=0$, we obtain (3.9).

The result for $\left(w^{0}, w^{1}\right) \in \mathcal{H}^{\prime}$ easily follows by a standard density argument.

Hence, we get the following moment problem.

Theorem 3.1. The initial datum $\left(u^{0}, u^{1}\right) \in \mathcal{H}$ is controllable if and only if there exists $v \in$ $L^{2}(0, T)$ such that

$$
\int_{a}^{b} \int_{0}^{T} v(t, x) e^{-i \nu_{n} t} d t d x=a_{n} \quad\left(n \in \mathbb{Z}^{*}\right)
$$

where $\nu_{n}=\operatorname{sgn}(n)|n|^{\alpha}$ verifies that $\left(i \nu_{n}\right)_{n \in \mathbb{Z}^{*}}$ are the eigenvalues of the elliptic operator associated to the continuous problem (3.10).

Proof of Theorem 3.1. Not that it is sufficient to show (3.9) is verified only for $\left(w^{0}, w^{1}\right)=$ $\Phi^{n}, n \in \mathbb{Z}^{*}$, where the eigenfunction of the fractional Laplacian operator is given by

$$
\Phi^{n}=\left(\frac{1}{n^{\alpha} i} \sin (n \pi x),-\sin (n \pi x)\right)^{T} .
$$

Hence, by considering $\left(w^{0}, w^{1}\right)=\Phi^{n}, n \in \mathbb{Z}^{*}$, in Lemma 3.1 we get

$$
\begin{gathered}
\int_{0}^{1} u^{0}(x) \bar{w}^{1}(x)-u^{1}(x) \bar{w}^{0}(x) d x=\frac{i a_{n}}{n^{\alpha}}, \\
\int_{a}^{b} \int_{0}^{T} v(t, x) \bar{w}(t, x) d t d x=\int_{a}^{b} \int_{0}^{T} \frac{i}{n^{\alpha}} v(t, x) e^{-i \nu_{n} t} d t d x,
\end{gathered}
$$

where for the last identity we have used the fact that $\bar{w}(t, x)=\frac{i e^{-i \nu_{n} t}}{n^{\alpha}}$. The proof is now finished combining the last two identities in Lemma 3.1.

Proof of Corollary 1. First of all, we remark that initial datum from (1.6) can be written as

$$
\left(u_{j}^{0}, u_{j}^{1}\right)_{1 \leq j \leq N}=\sum_{1 \leq|n| \leq f(N)} a_{n}(h) \Phi_{h}^{n}
$$

where $a_{n}(h)= \begin{cases}\frac{1}{2}\left(\frac{\lambda_{n}}{(n \pi)^{\alpha}}+1\right) a_{n}+\frac{1}{2}\left(\frac{\lambda_{n}}{(n \pi)^{\alpha}}-1\right) a_{-n} & |n| \leq f(N), \\ 0 & |n|>f(N) .\end{cases}$

The sequence of uniformly bounded controls $\left(v_{h}\right)_{h>0}$ given by Theorem 1.1 satisfies the discrete moment problem (2.7), which gives

$$
h \sum_{1 \leq j \leq N}(\sin (j \pi n h)) \chi_{a, b}(j h) \int_{0}^{T} e^{-i \lambda_{n} t} v_{h}(t, j h) d t=a_{n}(h) \quad(1 \leq|n| \leq N) .
$$

Let us consider a weak limit (up to a subsequence) $v$ of the discretized controls $\left(v_{h}\right)_{h>0}$. 
Note that for any $n \in \mathbb{Z}^{*}$, we have

$$
\begin{gathered}
h \sum_{1 \leq j \leq N}(\sin (j \pi n h)) \chi_{a, b}(j h) \int_{0}^{T} e^{-i \lambda_{n} t} v_{h}(t, j h) d t \underset{h \rightarrow 0}{\longrightarrow} \int_{a}^{b} \int_{0}^{T} \sin (n \pi x) e^{-i \lambda_{n} t} v(t, x) d t d x, \\
a_{n}(h) \underset{h \rightarrow 0}{\longrightarrow} a_{n} .
\end{gathered}
$$

Finally, if we combine the above estimates with the continuous moment problem (3.11), it follows that any weak limit $v$ of a subsequence of controls $\left(v_{h}\right)_{h>0}$ verifies

$$
\int_{a}^{b} \int_{0}^{T} \sin (n \pi x) e^{-i \lambda_{n} t} v(t, x) d t d x=a_{n} \quad\left(n \in \mathbb{Z}^{*}\right)
$$

hence, $v$ is a control for continuous problem (1.1).

\section{A Appendix: Proof of Theorem 1.2}

Assume by contradiction that for any $\left(u_{j}^{0}, u_{j}^{1}\right)_{1 \leq j \leq N}=\sum_{1 \leq|n| \leq N} a_{n} \Phi_{h}^{n}$ there exists a uniformly bounded sequence of controls $\left(v_{h}\right)_{h>0}$ for the discrete control problem (1.3) verifying that for any $h>0$, the solution $\left(u_{1}, \ldots u_{N}\right)$ of $(1.3)$ verifies $\left(u_{1}(T), \ldots, u_{N}(T)\right)=(0, \ldots, 0)$. By duality, this is totally equivalent to proving the following observability inequality: there exists $C>0$ (independent on $h>0$ ) such that for any

$$
Z^{0}=\sum_{1 \leq|n| \leq N} a_{n}^{0} \Phi_{h}^{n}
$$

then the solution of system (2.2) given by

$$
Z(t)=\sum_{1 \leq|n| \leq N} a_{n}^{0} e^{i \lambda_{n} t} \Phi_{h}^{n}
$$

verifies

$$
E(h)=\frac{h}{2} \sum_{1 \leq|n| \leq N}\left|a_{n}^{0}\right|^{2} \leqslant C \int_{0}^{T} \sum_{1 \leq|n| \leq N} \|\left. B_{h}^{n} a_{n}^{0} e^{i \lambda_{n} t}\right|^{2} d t,
$$

where the definition of the discrete energy $E$ is given in (2.3) and the definition of the discrete observation $B_{h}^{n}$ is given in (3.2). Let us consider the following initial condition:

$$
Z^{0}=\Phi_{h}^{N}-\Phi_{h}^{N-1}
$$

Then, inequality (A.1) becomes

$$
\begin{aligned}
h & \leqslant C \int_{0}^{T}\left\|B_{h}^{N} a_{N}^{0} e^{i \lambda_{N} t}-B_{h}^{N-1} a_{N-1}^{0} e^{i \lambda_{N-1} t}\right\|^{2} d t \\
& \leqslant C \int_{0}^{T} h^{2} \sum_{j=\left\lceil\frac{a}{h}\right\rceil}^{\left\lfloor\frac{b}{h}\right\rfloor}\left(e^{i \lambda_{N} t} \sin (j \pi N h)-e^{i \lambda_{N-1} t} \sin (j \pi(N-1) h)\right)^{2} d t \\
& \leqslant C h \int_{0}^{T}\left(h \sum _ { j = \lceil \frac { a } { h } \rceil } ^ { \lfloor \frac { b } { h } \rfloor } \left(|\sin (j \pi N h)|^{2}+|\sin (j \pi(N-1) h)|^{2}\right.\right. \\
& \left.\left.\left.-2 \cos \left(\left(\lambda_{N}-\lambda_{N-1}\right) t\right)\right) \sin (j \pi N h) \sin (j \pi(N-1) h)\right)\right) d t .
\end{aligned}
$$


Straightforward computations give that:

$$
\begin{aligned}
& h \sum_{j=\left\lceil\frac{a}{h}\right\rceil}^{\left\lfloor\frac{b}{h}\right\rfloor}|\sin (j \pi N h)|^{2} \rightarrow \frac{b-a}{2}+\frac{\sin (2 a \pi)-\sin (2 b \pi)}{4 \pi} \text { as } h \rightarrow 0, \\
& h \sum_{j=\left\lceil\frac{a}{h}\right\rceil}^{\left\lfloor\left\lfloor\frac{b}{h}\right\rfloor\right.}|\sin (j \pi(N-1) h)|^{2} \rightarrow \frac{b-a}{2}+\frac{\sin (2 a \pi)-\sin (2 b \pi)}{4 \pi} \text { as } h \rightarrow 0, \\
& \left.h \sum_{j=\left\lceil\frac{a}{h}\right\rceil}^{\left\lfloor\frac{b}{h}\right\rfloor} \sin (j \pi N-1) h\right) \sin (j \pi N h) \rightarrow \frac{b-a}{2}+\frac{\sin (2 a \pi)-\sin (2 b \pi)}{4 \pi} \text { as } h \rightarrow 0 .
\end{aligned}
$$

Moreover, it is very easy to prove that $\lambda_{N}-\lambda_{N-1} \rightarrow 0$ as $h \rightarrow 0$ (see Remark 1.1), so that by the dominated convergence Theorem, $\left.\int_{0}^{T} \cos \left(\left(\lambda_{N}-\lambda_{N-1}\right) t\right)\right) d t \rightarrow T$ as $h \rightarrow 0$. Hence, dividing by $h$ and making $h \rightarrow 0$ in (A.2) leads to a contradiction since the left-hand side is equal to 1 whereas the right-hand side tends to 0 as $h \rightarrow 0$.

\section{References}

[1] Amblard, F., Maggs, A.C., Yurke, B., Pargellis, A.N. and Leibler, S., Subdiffusion and Anomalous Local Viscoelasticity in Actin Networks, Phys. Rev. Lett. 77 (1996) 4470.

[2] Ammar-Khodja, F., Benabdallah, A., ,González-Burgos, M. and Morancey, M., Quantitative Fattorini-Hautus test and minimal null control time for parabolic problems. Preprint, 2017.

[3] Allonsius, D., Boyer, F. and Morancey, M., Spectral analysis of discrete elliptic operators and applications in control theory, Preprint, 2016.

[4] Biccari, U., Internal control for non-local Schrödinger and wave equations involving the fractional Laplace operator, to appear at ESAIM:COCV.

[5] Biccari, U. and Hernandez-Santamaria, V., Controllability of a one-dimensional fractional heat equation: theoretical and numerical aspects, to appear at IMA Journal of Mathematical Control and Information.

[6] Bugariu, I. F., Micu, S., and Rovenţa, I., Approximation of the controls for the beam equation with vanishing viscosity, Math. Comp. 85 (2016), 2259-2303.

[7] Cannarsa, P., Martinez, P. and Vancostenoble, J., The cost of controlling weakly degenerate parabolic equations by boundary controls. Math. Control Relat. Fields, 7(2) (2017), 171-211.

[8] Carcione, J. M., A generalization of the Fourier pseudospectral method, Geophysics, 75, no. 6, (2010), A53-A56.

[9] Castro, C. and Micu, S., Boundary controllability of a linear semi-discrete 1-D wave equation derived from a mixed finite element method, Numer. Math. 102 (2016), no. 3, 413-462.

[10] Chen, H., H. Zhou, and Qu, S., Lowrank approximation for time domain viscoacoustic wave equation with spatially varying order fractional Laplacians, 84th Annual International Meeting, SEG (2014).

[11] Chen, W., and S. Holm, Fractional Laplacian time-space models for linear and nonlinear lossy media exhibiting arbitrary frequency power-law dependency, The Journal of the Acoustical Society of America, 115 (2004), 14241430

[12] Cîndea, N., Micu, S. and Tucsnak, M., An approximation method for exact controls of vibrating systems, SIAM J. Control Optim. 49 (2011), no. 3, 1283-1305.

[13] Cîndea, N., Micu, S. and Rovenţa, I., Boundary controllability for finite-differences semidiscretizations of a clamped beam equation, SIAM J. Control Optim. 55 (2017), no. 2, 785-817.

[14] Dubkov, A. A., Spagnolo, B. and Uchaikin, V. V., Levy superflight diffusion: an introduction, Int. J. Bifurcation Chaos 18, 2649-2672 (2008).

[15] Ervedoza, S., Zheng C. and Zuazua, E., On the observability of time-discrete conservative linear systems, J. Funct. Anal. 254 (2008), no. 12, 3037-3078. 
[16] Ervedoza, S., Spectral conditions for admissibility and observability of wave systems: applications to finite element schemes, Numer. Math., 113 (2009), 377-415.

[17] Ervedoza, S., Observability in arbitrary small time for discrete conservative linear systems, Some Problems in Nonlinear Hyperbolic Equations, ed. Tatsien Li, Yuejun Peng and Bopeng Rao, Series in Contemporary Mathematics CAM15, 283-309.

[18] Ervedoza, S., Observability properties of a semi-discrete $1 D$ wave equation derived from a mixed finite element method on nonuniform meshes, ESAIM Control Optim. Calc. Var. 16 (2010), no. 2, 298-326.

[19] Ervedoza, S., Marica, A. and Zuazua, E., Numerical meshes ensuring uniform observability of one-dimensional waves: construction and analysis, IMA J. Numer. Anal. 36 (2016), no. 2, 503-542.

[20] Fattorini, H. O. and Russell, D. L.,Exact controllability theorems for linear parabolic equations in one space dimension, Archive for Rational Mechanics and Analysis, 43 (1971), 272-292.

[21] Glowinski, R., Li, C. H., and Lions, J.-L., A numerical approach to the exact boundary controllability of the wave equation (I). Dirichlet controls: Description of the numerical methods, Japan J. Appl. Math., 7 (1990), $1-76$.

[22] R. Kimmich, NMR: Tomography, Diffusometry, Relaxometry, Springer, Berlin, 1997.

[23] Komornik, V., and Loreti, P., Fourier Series in Control Theory, Springer-Verlag, New-York, 2005.

[24] Lagnese, J., Control of wave processes with distributed controls supported on a subregion, SIAM J. Control Optim. 21 (1983), no. 1, 68-85.

[25] Leon, L., and Zuazua, E., Boundary controllability of the finite-difference space semi-discretizations of the beam equation, ESAIM Control Optim. Calc. Var, A Tribute to J.-L. Lions, Tome 2 (2002), 827-862.

[26] Lissy, P., and Rovenţa, I., Optimal filtration for the approximation of boundary controls for the one-dimensional wave equation using finite-difference method, Math. Comp., (2018), DOI: https://doi.org/10.1090/mcom/3345.

[27] Lissy, P., On the cost of fast controls for some families of dispersive or parabolic equations in one space dimension, SIAM J. Control Optim. 52 (2014), no. 4, 2651-2676.

[28] Lissy, P., Explicit lower bounds for the cost of fast controls for some 1-D parabolic or dispersive equations, and a new lower bound concerning the uniform controllability of the 1-D transport-diffusion equation, J. Differential Equations 259 (2015), no. 10, 5331-5352.

[29] Lissy, P., Construction of Gevrey functions with compact support using the Bray-Mandelbrojt iterative process and applications to the moment method in control theory, Mathematical Control and Related Fields, Volume 7, Issue 1, March 2017, 21-40.

[30] Metzler, R., and J. Klafter, J., The random walk's guide to anomalous diffusion: A fractional dynamics approach, Phys. Rep., 339 (2000), 1-77.

[31] Micu, S., Uniform boundary controllability of a semi-discrete 1-D wave equation, Numer. Math. 91 (2002), 723-768.

[32] Micu, S., Uniform boundary controllability of a semidiscrete 1-D wave equation with vanishing viscosity, SIAM J. Control Optim. 47 (2008), no. 6, 2857-2885.

[33] Micu, S. and Zuazua, E., On the controllability of a fractional order parabolic equation. (English summary), SIAM J. Control Optim. 44 (2006), no. 6, 1950-1972.

[34] Micu, S., Rovenţa, I. and Temereanca, L. E., Approximation of the controls for the linear beam equation, Math. Control Signals Systems 28 (2016), no. 2, Art. 12, 53 pp.

[35] Miller, L., On the controllability of anomalous diffusions generated by the fractional Laplacian, Math. Control Signals Systems 18 (2006), no. 3, 260-271.

[36] Miller, L., Resolvent conditions for the control of unitary groups and their approximations, Journal of Spectral Theory 2 (2012), 1-55.

[37] Münch, A., Famille de schemas implicites uniformement controlables pour l'equation des ondes 1-D. (French.English, French summary) [Family of implicit schemes uniformly controllable for the 1-D wave equation], C. R. Math. Acad. Sci. Paris 339 (2004), no. 10, 733-738.

[38] Porto, M., Bunde, A. , Havlin, S. and Roman, H.E., Probability densities for the displacement of random walks on percolation clusters, Phys. Rev. E 56 (1997) 1667.

[39] Richardson, L.F., Atmospheric diffusion shown on a distance-neighbour graph, Proc. Roy. Soc. 110 (1926) 709-737. 
[40] Rudin, W., Real and complex analysis. Third edition, McGraw-Hill Book Co., New York, 1987. xiv+416 pp.

[41] Scher, H. and Montroll, E.W., Anomalous transit-time dispersion in amorphous solids, Phys. Rev. B 12 (1975) 2455.

[42] Servadei, R. and Valdinoci, E., On the spectrum of two different fractional operators, Proceedings of the Royal Society of Edinburgh Section A: Mathematics, Volume 144, Issue 4 August 2014, 831-855.

[43] Sokolov, I., Klafter, J. and Blumen, A., Fractional kinetics, Physics Today 55 (2002), 48-54.

[44] Sun, J., Zhu T. and Fomel, S., Viscoacoustic modeling and imaging using low-rank approximation, 84th Annual International Meeting, SEG (2014).

[45] Szekeres, B. and Izsák, F., Finite difference approximation of space-fractional diffusion problems: the matrix transformation method. (English summary), Comput. Math. Appl. 73 (2017), no. 2, 261-269.

[46] Treeby, B. E. and Cox, B., Modeling power law absorption and dispersion for acoustic propagation using the fractional Laplacian The Journal of the Acoustical Society of America, 127 (2010), 2741- 2748,

[47] Yao, J., Zhou, T., Hussain F. and Kouri, D. J., Solving fractional Laplacian viscoacoustic wave equation using Hermite distributed approximating functional method, 86th Annual International Meeting, SEG (2016).

[48] Yuan, Q.-Y. and Ma, Z.-M., Boundary Problems for Fractional Laplacians, Stoch. Dyn. 5 (2005), 385-424.

[49] Zuazua, E., Propagation, observation, and control of waves approximated by finite difference methods, SIAM Rev., 47 (2005), 197-243 (electronic).

[50] Zheng, G. and Ali, M. M., Observability estimate for the fractional order parabolic equations on measurable sets, Abstr. Appl. Anal. 2014, Art. ID 361904, 5 pp.

[51] Zhu, T., Carcione, J. M. and Harris, J. M., Approximating constant-Q seismic propagation in the time domain Geophysical Prospecting, 61 (2014), 931-940.

[52] Zhu, T. and Harris, J. M., Modeling acoustic wave propagation in heterogeneous attenuating media using decoupled fractional Laplacians, Geophysics, 79, no. 3, T105-T116 (2013). 